\title{
Persistence probabilities and exponents
}

\author{
Frank Aurzada and Thomas Simon
}

\begin{abstract}
This article deals with the asymptotic behavior as $t \rightarrow+\infty$ of the survival function $\mathbb{P}[T>t]$, where $T$ is the first passage time above a non negative level of a random process starting from zero. In many cases of physical significance, the behavior is of the type $\mathbb{P}[T>t]=t^{-\theta+o(1)}$ for a known or unknown positive parameter $\theta$ which is called the persistence exponent. The problem is well understood for random walks or Lévy processes but becomes more difficult for integrals of such processes, which are more related to physics. We survey recent results and open problems in this field.
\end{abstract}

\section{AMS Subject Classification 2000:}

60F99, 60G10, 60G15, 60G18, 60G50, 60G52, 60K35, 60K40

Key words: First passage time, Gaussian process, integrated process, Lévy process, lower tail probability, persistence, random walk, stable process

\section{Introduction}

Let $\left\{X_{t}, t \geq 0\right\}$ be a real stochastic process in discrete or continuous time, starting from zero. The analysis of the first passage time $T_{x}=\inf \left\{t>0, X_{t}>x\right\}$ above a nonnegative level $x$ is a classical issue in probability. In this paper we will be concerned with the asymptotic behavior as $t \rightarrow+\infty$ of the survival function $\mathbb{P}\left[T_{x}>t\right]$ for a

Frank Aurzada

Technische Universität Darmstadt, Fachbereich Mathematik, Schlossgartenstr. 7, D-64289 Darmstadt. e-mail: aurzada@mathematik.tu-darmstadt. de

Thomas Simon

Laboratoire Paul Painlevé, Université de Lille 1, Cité Scientifique, F-59655 Villeneuve d'Ascq Cedex. Laboratoire de Physique Théorique et Modèles Statistiques, Université Paris Sud, Bâtiment 100, F-91405 Orsay Cedex. e-mail: simon@math. univ-lillel.fr 
class of processes related to random walks and Lévy processes. This problem has attracted some interest in the recent literature under the denomination persistence probability. In a self-similar framework, that is if $\left\{X_{c t}, t \geq 0\right\}$ and $\left\{c^{H} X_{t}, t \geq 0\right\}$ have the same distribution for some $H>0$, the question is equivalent to the lower tail probability problem, which is the study of the quantity

$$
\mathbb{P}\left[\sup _{0 \leq s \leq 1} X_{s}<x\right], \quad \text { as } x=t^{-H} \rightarrow 0 .
$$

In many situations of interest it turns out that the behavior is polynomial: one has

$$
\mathbb{P}\left[T_{x}>t\right]=t^{-\theta+o(1)}
$$

for a non-negative parameter $\theta$ called the persistence exponent, which usually does not depend on $x \geq 0$ and often belongs to $[0,1]$. The study of asymptotic behaviors like (1.1) has gained some attraction over the last years in the physics literature as well, where the parameter $\theta$ is often called survival exponent. Estimates of the type (1.1) also appear in reliability theory, a subject that we shall not discuss here see [44] and the references therein for an account. In the following we will mostly be concerned with positive persistence exponents, that is the process ends up in crossing the level $x$. This contrasts sharply with classical risk theory, where the survival analysis deals with the probability for a given process never to cross a fixed level - see [1].

If $X$ is a random walk or a Lévy process, studying the law of $T_{x}$ is a special part of fluctuation theory and the persistence probabilities are then well understood. For example if $X_{1}$ is attracted to a stable law, classical fluctuation identities entail that the persistence exponent is the positivity parameter of the latter. There are many accounts on fluctuation theory and we refer in particular to [18] for random walks and to [39] for Lévy processes. In the first part of this article we focus on the results of this theory dealing with the asymptotic behavior of $\mathbb{P}\left[T_{x}>t\right]$, and we try to be as exhaustive as possible. In general, the behavior is the same in discrete and continuous time, except of course for $x=0$ where the problem becomes different for Lévy processes. We notice that even though a posteriori the resulting exponents turn out to be the same for a Lévy process and the respective random walk, there is no simple approximation argument that would yield this a priori, and for this reason we have to consider the discrete and continuous time situations separately - a feature that we encounter also for more complicated processes. The results for random walks and Lévy processes, all classical, are presented here in order to give some insight into more complex situations where $X$, though constructed upon a Lévy process or a random walk, is not a Markov process anymore.

Some of these more complex situations, which are called non-trivial in the physical literature, are the matter of the second part of this article. We first consider integrated random walks and integrated Lévy processes. It turns out that for such simple constructions, the computation of the persistence exponent is not quite easy in general. We display recent and less recent results where the persistence probabilities are estimated with various degrees of precision, and which all have the common 
feature that the persistence exponent of the underlying process is twice the persistence exponent of its integral. We believe that this is a kind of universal rule and several conjectures are stated in this direction, the most general being Conjecture 2 and Conjecture 4. A curious fact is that the situation with no positive jumps - where classical fluctuation theory becomes much simpler - appears to be more difficult than the dual situation with no negative jumps, where some exponents of integrated processes can be computed. The reason for that seems to be only methodological, and to cast out the curse of spectral positivity in the study of persistence probabilities of integrated processes probably requires other, say deeper, tools.

We then consider fractionally integrated processes, where the situation is yet more difficult. The case when the underlying Lévy process is Brownian motion yields self-similar Gaussian processes which can be changed into the respective stationary ones by the Lamperti transformation. The sought after persistence exponent is then directly related to an estimate on the probability of non-zero crossings for these Lamperti transforms, a problem known to be hard in spite of the Itô-Rice formula which gives some information at the expectation level in the smooth case. We present some universality and monotonicity results, and also some partially heuristic comparisons with the fractional Brownian motion case, whose persistence exponent can be computed explicitly. We also display other explicit computations of persistence exponents for related processes such as weighted random walks, iterated processes, and autoregressive processes.

The third and last part of this article deals with some applications of the persistence probabilities in mathematical physics. We first deal with Lagrangian regular points of the inviscid Burgers equation with random self-similar initial data. The link between the Hausdorff dimension of such points and the persistence exponent of integrated processes dates back to the original paper [83]. We recall here that the problem is still open in the fractional Brownian motion case and state a plausible conjecture when the initial data is a two-sided stable Lévy process. Second, we consider the zero-crossings of a peculiar Gaussian stationary process which is related to the positivity of Kac polynomials with large even degree. This connection, which was discovered in [31], has further ramifications with the persistence exponents of integrals of Brownian motion with higher order and of a certain diffusion equation with white noise initial conditions in the plane, and we make a brief account on the subject. We last consider three different interacting statistical systems whose analysis hinges significantly upon the persistence probabilities of integrated processes: wetting models with Laplacian interaction, fluctuating interfaces with Langevin dynamics, and sticky particles on the line with Poissonian initial conditions.

Some open problems stated in the present paper are believed to be challenging and we think that they could catch the attention of some colleagues. We finally point out that we have not exhausted here all implications of persistence in physics and that the persistence exponent of many other models remains unknown - see $[27,21,66,63]$ and the references therein. 


\section{Classical results}

\subsection{Random walks}

Let $\left\{X_{n}, n \geq 1\right\}$ be a sequence of i.i.d. real random variables with common distribution $\mu$, and $S_{n}=X_{1}+\ldots+X_{n}$ be the associated random walk. Consider

$$
T_{x}=\inf \left\{n \geq 1, S_{n}>x\right\}
$$

the first-passage time above $x \geq 0$. Recall the following basic rule, to be found in e.g. Chapter XII.1-2 of [43]:

$$
T_{x} \text { is a.s. finite for every } x \geq 0 \Leftrightarrow T_{0} \text { is a.s. finite, }
$$

and that the latter is also equivalent to the fact that $S_{n}$ does not drift to $-\infty$. In this case one has $\mathbb{P}\left[T_{x}>n\right] \rightarrow 0$ as $n \rightarrow+\infty$ for every $x \geq 0$, and the difficulty to estimate the speed of convergence comes from the fact that the event $\left\{T_{x}>n\right\}=\left\{S_{1} \leq x, \ldots, S_{n} \leq x\right\}$ depends on $n$ correlated random variables.

If $\mu$ is concentrated on $\mathbb{R}^{+}$, then $\mathbb{P}\left[T_{x}>n\right]=\mathbb{P}\left[S_{n}<x\right]$ and the problem becomes one-dimensional. The straightforward inequality

$$
\mathbb{P}\left[S_{n}<x\right] \leq e^{x+n \log \left(\mathbb{E}\left[e^{-X_{1}}\right]\right)}
$$

shows that $\mathbb{P}\left[T_{x}>n\right]$ tends to zero at least exponentially fast (unless $\mu$ is degenerate at zero). Notice that the rate might also be superexponential and depend on $x$ at the logarithmic scale: if $X_{1}$ has a positive strictly $\alpha$-stable law $(0<\alpha<1)$ for instance, de Bruijn's Tauberian theorem - see Theorem 4.12.9 in [19] - leads to

$-\log \mathbb{P}\left[T_{x}>n\right]=-\log \mathbb{P}\left[S_{n}<x\right]=-\log \mathbb{P}\left[S_{1}<x n^{-1 / \alpha}\right] \sim \kappa_{\alpha} x^{-\alpha /(1-\alpha)} n^{1 /(1-\alpha)}$

for every $x>0$, with some explicit $\kappa_{\alpha}>0$ - see also [89]. Here and below $a_{n} \sim b_{n}$ is defined by $\lim _{n \rightarrow \infty} a_{n} / b_{n}=1$ and analogously for other limits. Of course, one has $T_{0}=1$ a.s. whenever $\mu$ does not charge 0 . If $\mu\{0\}>0$, Jain and Pruitt's general uniform results on renewal sequences express the asymptotic behavior of $\mathbb{P}\left[S_{n}<x\right]$ in terms of quantities related to $\mu$, at the logarithmic scale - see Theorem 2.1 in [52] - and also at the exact scale, under some conservativeness assumption - see Theorem 4.1 in [52].

If $\mu$ is not concentrated on $\mathbb{R}^{+}$, it is easy to see that the asymptotics of $\mathbb{P}\left[T_{x}>n\right]$ will not drastically depend on $x$ : choosing $\varepsilon>0$ such that $\mu_{\varepsilon}=\mu(-\infty,-\varepsilon)>0$, the Markov property entails $\mathbb{P}\left[T_{x}>n\right] \geq \mathbb{P}\left[T_{0}>n\right] \geq \mathbb{P}\left[T_{\varepsilon}>n-1\right] \mathbb{P}\left[X_{1}<-\varepsilon\right] \geq$ $\mu_{\varepsilon} \mathbb{P}\left[T_{\varepsilon}>n\right] \geq \mu_{\varepsilon}^{k} \mathbb{P}\left[T_{x}>n\right]$ as soon as $k \varepsilon \geq x$, so that

$$
\mathbb{P}\left[T_{x}>n\right] \asymp \mathbb{P}\left[T_{0}>n\right]
$$


for every $x \geq 0$. Here and below $a_{n} \asymp b_{n}$ is defined by $0<\liminf _{n \rightarrow \infty} a_{n} / b_{n}$ and $\lim \sup _{n \rightarrow \infty} a_{n} / b_{n}<\infty$ and analogously for other limits.

The following formula computes the generating function of $\left\{\mathbb{P}\left[T_{0}>n\right], n \geq 0\right\}$ in terms of that of the sequence $\left\{n^{-1} \mathbb{P}\left[S_{n} \leq 0\right], n \geq 1\right\}$, and is true for any random walk. It is a cornerstone for further investigations.

Sparre Andersen's formula. For every $z \in]-1,1[$ one has

$$
\sum_{n \geq 0} z^{n} \mathbb{P}\left[T_{0}>n\right]=\exp \left[\sum_{n \geq 1} \frac{z^{n}}{n} \mathbb{P}\left[S_{n} \leq 0\right]\right] .
$$

This result is obtained after simple rearrangements from Theorem XII.7.1 in [43], whose proof has a combinatorial character and depends heavily on the independence and stationarity of the increments of the random walk. A simpler method relying on elementary Fourier analysis - see Chapter 3.7 in [40] - yields the more general

Spitzer's formula. For every $z \in]-1,1[$ and $\lambda \geq 0$, one has

$$
\sum_{n \geq 0} z^{n} \mathbb{E}\left[e^{-\lambda M_{n}}\right]=\exp \left[\sum_{n \geq 1} \frac{z^{n}}{n} \mathbb{E}\left[e^{-\lambda S_{n}^{+}}\right]\right]
$$

with the notation $M_{n}=\max \left(0, S_{1}, \ldots, S_{n}\right)$ and $S_{n}^{+}=\max \left(0, S_{n}\right)$.

Indeed, one obtains (2.3) as a consequence of (2.4) in letting $\lambda \rightarrow+\infty$. It is beyond the peculiar scope of the present paper to discuss the full strength and the various generalisations of Spitzer's formula such as Baxter-Spitzer's formula or the Wiener-Hopf factorization, and we refer e.g. to [18, 39, 43] for more on this subject. Let us simply remark that Sparre Andersen's formula entails easily - see e.g. Theorem XII.7.2 in [43] - the following characterization of (2.1):

$$
S_{n} \text { drifts to }-\infty \Leftrightarrow \sum_{n \geq 1} \frac{1}{n} \mathbb{P}\left[S_{n}>0\right]<+\infty \text {. }
$$

From now on, we will suppose that $S_{n}$ does not drift to $-\infty$ and that $\mu$ is not concentrated on $\mathbb{R}^{+}$. This entails that $\mathbb{P}\left[S_{n}>0\right]>0$ and $\mathbb{P}\left[S_{n}<0\right]>0$ for every $n \geq 1$.

A remarkable consequence of (2.3) is that when $\mathbb{P}\left[S_{n} \leq 0\right]=\rho \in(0,1)$ for every $n \geq 1$, one obtains an explicit formula for $\mathbb{P}\left[T_{0}>n\right]$ depending only on $\rho$ and $n$ : one has

$$
\sum_{n \geq 0} z^{n} \mathbb{P}\left[T_{0}>n\right]=\frac{1}{(1-z)^{\rho}}=\sum_{n \geq 0} \frac{\Gamma(n+\rho)}{n ! \Gamma(\rho)} z^{n}
$$

so that 


$$
\mathbb{P}\left[T_{0}>n\right]=\frac{\Gamma(n+\rho)}{n ! \Gamma(\rho)} \sim \frac{n^{\rho-1}}{\Gamma(\rho)} .
$$

For example, symmetric random walks such that $\mathbb{P}\left[S_{n}=0\right]=0$ for every $n \geq 1$ (this latter property is true when $\mu$ is non atomic, for instance) all enjoy the property that

$$
\mathbb{P}\left[T_{0}>n\right]=\frac{\Gamma(n+1 / 2)}{\sqrt{\pi} n !} \sim \frac{1}{\sqrt{\pi n}} .
$$

Recall in passing that the estimate is slightly different for the simple random walk where $\mu\{1\}=\mu\{-1\}=1 / 2$, since then $\mathbb{P}\left[S_{2 n}=0\right] \neq 0$. The classical computation, to be found e.g. at the beginning of the monograph [75]

$$
\begin{aligned}
\sum_{n \geq 0} z^{n} \mathbb{P}\left[T_{0}>n\right] & =\exp \left[\sum_{n \geq 1} \frac{z^{n}}{n} \frac{1}{2}-\sum_{n \geq 1} \frac{z^{2 n}}{2 n 2^{2 n}}\left(\begin{array}{c}
2 n \\
n
\end{array}\right)\right] \\
& =\left(\frac{1}{1-z}-\frac{1-\sqrt{1-z^{2}}}{z(1-z)}\right) \sim \sqrt{\frac{2}{1-z}} \text { as } z \uparrow 1,
\end{aligned}
$$

and the Tauberian theorem for monotonic sequences entail $\mathbb{P}\left[T_{0}>n\right] \sim \sqrt{2 /(\pi n)}$.

Another remarkable consequence of (2.4) is the exact computation of the persistence exponent whenever $\left\{S_{n}, n \geq 1\right\}$ fulfils the so-called Spitzer's condition

$$
\lim _{n \rightarrow \infty} \frac{1}{n} \sum_{k=1}^{n} \mathbb{P}\left[S_{k}<0\right]=\rho \in[0,1] .
$$

The latter turns out to be equivalent - see [15, 37], or Chapter 7 in [39] - to

$$
\lim _{n \rightarrow \infty} \mathbb{P}\left[S_{n}<0\right]=\rho,
$$

and a theorem of Rogozin shows that (2.6) entails

$$
\mathbb{P}\left[T_{x}>n\right] \sim \frac{c_{x} n^{\rho-1} l(n)}{\Gamma(\rho)}=n^{\rho-1+o(1)}
$$

for every $x \geq 0$ with $l(n)$ some slowly varying sequence and $c_{x}$ some explicit positive constant. Besides, as explained in Theorem 8.9.12 of [19], this asymptotic behavior is actually equivalent to (2.6) for $\rho \in(0,1)$. When $\mathbb{P}\left[S_{n}<0\right] \rightarrow 0$ various behaviors are possible, contrary to the above. For example if $n^{\rho} \mathbb{P}\left[S_{n}<0\right]$ is slowly varying for some $\rho \in(0,1)$, Theorem 8.9.14 in [19] and Theorem XVII.5.1 in [43] yield

$$
\mathbb{P}\left[T_{0}>n\right] \sim l(n) n^{-(1+\rho)}
$$

with $l(n)$ some slowly varying sequence. Other various behaviors also appear when $\mu$ has positive expectation and we refer to [38] for precise results. When $\mathbb{P}\left[S_{n}<0\right] \rightarrow 1$ and $S_{n}$ does not drift to $-\infty$ (that is, when $\left.\sum_{n}\left(1-\mathbb{P}\left[S_{n} \leq 0\right]\right) / n=\infty\right)$, 
the behavior of $\mathbb{P}\left[T_{0}>n\right]$ does not seem to be known, although one might expect that the persistence exponent is always zero.

We conclude this paragraph with the so-called upward skip-free or right-continuous random walks on $\mathbb{Z}$ viz. such that Supp $\mu \subseteq\{1,0,-1,-2,-3, \ldots\}$, for which our survival analysis does not require the use of (2.3). Indeed, the distribution of $T_{k}$ is then given by Kemperman's formula [54] which reads:

$$
\mathbb{P}\left[T_{k}=n\right]=\frac{k+1}{n} \mathbb{P}\left[S_{n}=k+1\right] \quad \text { for every } n>k .
$$

In this particular case, we are reduced to the asymptotical behavior of the onedimensional probability $\mathbb{P}\left[S_{n}=1\right]$. Notice - see [55] - that there is a universal upper bound $\mathbb{P}\left[S_{n}=1\right] \leq c n^{-1 / 2}$ leading to $\mathbb{P}\left[T_{k}>n\right] \leq 2 c(k+1) n^{-1 / 2}$, but the exact behavior of $\mathbb{P}\left[S_{n}=1\right]$ depends on $\mu$. For example, if $\mu$ is in the respective domain of normal attraction of some strictly $\alpha$-stable law - with $\alpha \in(1,2]$ since otherwise $S_{n}$ would drift to $-\infty$ by the law of large numbers, Gnedenko's local limit theorem see e.g. Theorem 8.4.1 in [19] - yields $\mathbb{P}\left[S_{n}=1\right] \sim c_{\alpha} n^{-1 / \alpha} l(n)$ for some explicit $c_{\alpha}$, so that by (2.9)

$$
\mathbb{P}\left[T_{k}>n\right] \sim \alpha c_{\alpha}(k+1) l(n) n^{-1 / \alpha} .
$$

Recall - see Proposition 8.9.16 in [19] and recall that we deal with right-continuous walks - that $\mu$ is in the domain of attraction of some strictly $\alpha$-stable law with $\alpha \in(1,2]$ if and only if (2.6) holds with $\rho=1-1 / \alpha$, so that (2.10) is also actually a consequence of (2.7). On the other hand, the slowly varying term $l(n)$ can be removed if $S_{n}$ is in the respective domain of normal attraction (viz. when $S_{n} / n^{1 / \alpha}$ converges in law to some non degenerate limit - see e.g. the concluding remark of Chapter XVII.5 in [43]), and this degree of precision is not given by Rogozin's theorem.

\subsection{Lévy processes}

Let $\left\{Z_{t}, t \geq 0\right\}$ be a non-degenerate real Lévy process starting from 0 and

$$
T_{x}=\inf \left\{t>0, Z_{t}>x\right\}
$$

be its first passage time above $x \geq 0$. Consider $\left\{Z_{n}, n \geq 1\right\}$ the associated random walk. The inequality

$$
\mathbb{P}\left[T_{x}>t\right] \leq \mathbb{P}\left[\tilde{T}_{x}>[t]\right]
$$

with the notation $\tilde{T}_{x}=\inf \left\{n \geq 1, Z_{n}>x\right\}$, yields a rough upper bound for $\mathbb{P}\left[T_{x}>t\right]$ which can be made more precise as a function of $t$ and $x$ in applying the results of the previous paragraph. This upper bound however does not yield enough information in general if $x=0$. For example Rogozin's criterion - see Proposition VI.11 in [13] 
and the remark thereafter - shows that $T_{0}=0$ a.s. when $Z$ has unbounded variations, whereas the function $t \mapsto \mathbb{P}\left[Z_{1} \leq 0, \ldots, Z_{[t]} \leq 0\right]$ has a positive limit at $+\infty$ if $Z$ drifts to $-\infty$. Recall also that in the bounded variation case, the regularity of the half-line for $Z$ is characterized in terms of the Lévy measure and the drift - see Theorem 22 in [39], and again $\mathbb{P}\left[Z_{1} \leq 0, \ldots, Z_{[t]} \leq 0\right]$ might have a positive limit when $t \rightarrow+\infty$ eventhough $T_{0}=0$ a.s.

If $x>0$ however, it turns out that the two quantities in (2.11) are often comparable. First of all, for every $x>0$ one has

$$
\mathbb{P}\left[T_{x}=+\infty\right]>0 \Leftrightarrow Z_{t} \rightarrow-\infty \text { a.s. } \Leftrightarrow \int_{1}^{\infty} \frac{1}{t} \mathbb{P}\left[Z_{t}>0\right] \mathrm{d} t<+\infty
$$

(see e.g. Corollary 4.4 (iii) in [39]), and a simple analysis shows that $Z_{t} \rightarrow-\infty$ a.s. is equivalent to $Z_{n} \rightarrow-\infty$ a.s. which is itself equivalent to

$$
\sum_{n \geq 1} \frac{1}{n} \mathbb{P}\left[Z_{n}>0\right]<+\infty
$$

so that with the above notation $\mathbb{P}\left[T_{x}>t\right] \rightarrow 0$ if and only if $\mathbb{P}\left[\tilde{T}_{x}>t\right] \rightarrow 0$ as $t \rightarrow+\infty$. In the following, we will see that in many examples one has

$$
\mathbb{P}\left[T_{x}>t\right] \asymp \mathbb{P}\left[\tilde{T}_{x}>[t]\right] .
$$

However, it does not seem easy to prove this estimate a priori, which probably does not hold in full generality.

We will suppose henceforth that (2.12) does not hold. Set $\mu$ for the law of $Z_{1}$, $M_{t}=\sup \left\{Z_{s}, s \leq t\right\}$ for the running maximum of $Z$, and recall that

$$
\mathbb{P}\left[T_{x}>t\right]=\mathbb{P}\left[M_{t} \leq x\right] .
$$

If $\mu$ is concentrated on $\mathbb{R}^{+}$then $Z$ is a.s. increasing and one is reduced to the random walk case because

$$
\mathbb{P}\left[Z_{[t]+1} \leq x\right] \leq \mathbb{P}\left[T_{x}>t\right]=\mathbb{P}\left[Z_{t} \leq x\right]=\mathbb{P}\left[M_{t} \leq x\right] \leq \mathbb{P}\left[Z_{[t]} \leq x\right] .
$$

If $\mu$ is not concentrated on $\mathbb{R}^{+}$then an argument analogous to that of the previous paragraph shows that

$$
\mathbb{P}\left[T_{x}>t\right] \asymp \mathbb{P}\left[T_{y}>t\right]
$$

as $t \rightarrow+\infty$ for every $x, y>0$, as in the discrete time framework. The classical approach to obtain more information on $\mathbb{P}\left[T_{x}>t\right]$ relies on a particular case of the first fluctuation identity - see e.g. Theorem VI.5 in [13], which is an analogue of Spitzer's formula in continuous time: 
Baxter-Donsker's formula. For every $\lambda \geq 0$ and $q>0$, one has

$$
\mathbb{E}\left[e^{-\lambda M_{e q}}\right]=\exp -\left[\int_{0}^{\infty} \frac{e^{-q t}}{t} \mathbb{E}\left[\left(1-e^{-\lambda Z_{t}^{+}}\right)\right] \mathrm{d} t\right]
$$

with the notation $Z_{t}^{+}=\max \left(0, Z_{t}\right)$ and $e_{q} \sim \operatorname{Exp}(q)$ an independent random time.

An important consequence of this formula is a theorem of Rogozin - see e.g. Theorem VI.18 in [13] - which shows that if the following Spitzer's condition

$$
\frac{1}{t} \int_{1}^{t} \mathbb{P}\left[Z_{s} \geq 0\right] d s \rightarrow \rho \in(0,1), \quad t \rightarrow+\infty
$$

holds, then for any $x>0$

$$
\mathbb{P}\left[T_{x}>t\right] \sim c_{x} l(t) t^{-\rho}
$$

with $c_{x}>0$ and $l(t)$ some slowly varying function not depending on $x$. Besides, one can show that (2.14) and (2.15) are actually equivalent - see again Theorem VI.18 in [13]. Recall - see Chapter 7 in [39] - that (2.14) is also equivalent to (2.6) for the random walk $\left\{-Z_{n}, n \geq 1\right\}$. The estimate (2.15) can be refined for strictly $\alpha$-stable processes, which all enjoy the property that

$$
\mathbb{P}\left[Z_{t} \geq 0\right]=\rho \in(0,1) \quad \text { for every } t>0 .
$$

An asymptotic analysis of the so-called Darling integral - see Theorem 3b in [16] entails then

$$
\mathbb{P}\left[T_{x}>t\right] \sim c x^{\alpha \rho} t^{-\rho}
$$

for such processes, where $c$ is an explicit constant. Notice that there are other Lévy processes enjoying the property (2.16), like subordinate stable processes, for which no refinement of (2.15) seems available in the literature. In [12], the precise estimate

$$
\mathbb{P}\left[T_{x}>t\right] \sim c_{x} t^{-1 / 2}
$$

was obtained for every centered Lévy processes with finite variance and every $x>0$. We finally remark that Section 3 in [57] provide uniform estimates for $\mathbb{P}\left[T_{x}>t\right]$ in terms of the renewal function of $Z$, the latter being non-explicit in general.

We conclude this paragraph with spectrally negative Lévy processes, where our survival analysis amounts to the study of a one-dimensional probability as in the discrete framework, and one does not really require (2.13). Indeed, the passagetime process $\left\{T_{x}, x \geq 0\right\}$ is then a subordinator with infinite lifetime if $Z$ does not drift to $-\infty$, whose Laplace exponent $\Phi(\lambda)=-\log \mathbb{E}\left[e^{-\lambda T_{1}}\right]$ is characterized by the law of $Z_{1}$ - see Chapter VII in [13] or Chapter 9 in [39] for these basic facts. In particular, if $\Phi(\lambda) \sim \lambda^{\rho} l(\lambda)$ as $\lambda \rightarrow 0$ for some $\rho \in(0,1)$ and $l(\lambda)$ some slowly varying function, then Theorem XIII.5.4. in [43] yields 


$$
\mathbb{P}\left[T_{x}>t\right] \sim \frac{x}{\Gamma(\rho)} l\left(t^{-1}\right) t^{-\rho}
$$

Actually, the above condition on $\Phi$ is equivalent to (2.14) - see Proposition VII.6 in [13] and notice that then necessarily $\rho \geq 1 / 2$. Hence, the above estimate is just a consequence of Rogozin's theorem with an explicit constant $c_{x}$. Spectrally negative Lévy processes also enjoy the following peculiar property, which follows easily from the strong Markov property and the absence of positive jumps:

$$
M_{e_{q}} \stackrel{d}{=} Z_{e_{q}} \mid Z_{e_{q}}>0
$$

In particular, one has $M_{t} \stackrel{d}{=} Z_{t} \mid Z_{t}>0$ for every $t>0$ if $\mathbb{P}\left[X_{t}>0\right]=\rho \geq 1 / 2$ does not depend on $t$ (which is true only for strictly $(1 / \rho)$-stable process). This latter identity which can be shown in many different ways - see Section 8 in [17] and the references therein - recovers the estimate $(2.17)$ in this particular case: one has

$$
\mathbb{P}\left[T_{x}>t\right] \sim \frac{c x}{\Gamma(\rho)} t^{-\rho}
$$

for some explicit constant $c>0$ which is $\sqrt{2}$ for the standard Brownian motion. We finally stress that (2.18) can be useful for spectrally negative processes such that $\mathbb{P}\left[X_{t}>0\right] \rightarrow 1$. For example, if $X$ is an $\alpha$-stable process with positive drift then (2.18) shows after a simple analysis that

$$
\mathbb{P}\left[T_{x}>t\right] \sim c x t^{-\alpha}
$$

for some explicit $c>0$, an estimate which cannot be obtained directly neither from Rogozin's theorem nor from the behavior of $\Phi$ at zero, and which is also coherent with (2.8) since $\mathbb{P}\left[X_{t}<0\right] \sim c t^{1-\alpha}$.

\section{Recent advances}

\subsection{Integrated random walks}

An integrated random walk is the sequence of partial sums $A_{n}=S_{1}+\cdots+S_{n}$, where $\left\{S_{n}, n \geq 1\right\}$ is a random walk. As above we write $S_{n}=X_{1}+\cdots+X_{n}$ and we denote by $\mu$ the law of the increment $X_{1}=S_{1}$. Let

$$
T_{x}=\inf \left\{n \geq 1, A_{n}>x\right\}
$$

be the first-passage time above $x \geq 0$. Since we have

$$
\left\{S_{1} \leq 0, \ldots, S_{n} \leq 0\right\} \subseteq\left\{A_{1} \leq 0, \ldots, A_{n} \leq 0\right\}
$$


the discussion made in Paragraph 2.1 shows that $\mathbb{P}\left[T_{x}=+\infty\right] \geq \mathbb{P}\left[T_{0}=+\infty\right]>0$ as soon as $S_{n}$ drifts to $-\infty$. If $\mu$ is concentrated on $\mathbb{R}^{+}$, then $A_{n}$ has non-negative increments and since $A_{n}=n X_{1}+(n-1) X_{2} \cdots+X_{n} \geq(n / 2) S_{[n / 2]}$, one has

$$
\mathbb{P}\left[T_{x}>n\right]=\mathbb{P}\left[A_{n}<x\right] \leq \mathbb{P}\left[n S_{[n / 2]}<2 x\right] \leq e^{2 x+n \log \left(\mathbb{E}\left[e^{-[n / 2] X_{1}}\right]\right)},
$$

which shows that $\mathbb{P}\left[T_{x}>n\right]$ tends to zero superexponentially fast, unless $\mu$ is degenerate. If $\mu$ is not concentrated on $\mathbb{R}^{+}$, then choosing $\varepsilon$ such that $\mu(-\infty,-\varepsilon)>0$ and using the same argument as in Paragraph 2.1 entail $\mathbb{P}\left[T_{x}>n\right] \geq \mathbb{P}\left[T_{0}>n\right] \geq$ $(\mu(-\infty,-\varepsilon))^{k} \mathbb{P}\left[T_{x}>n\right]$ for every $x \geq 0$ as soon as $k \varepsilon \geq x$, so that

$$
\mathbb{P}\left[T_{x}>n\right] \asymp \mathbb{P}\left[T_{0}>n\right]
$$

for every $x \geq 0$.

Henceforth we will suppose that $S_{n}$ does not drift to $-\infty$ and that $\mu$ is not concentrated on $\mathbb{R}^{+}$. This entails that $\mathbb{P}\left[S_{n}>0\right]>0$ and $\mathbb{P}\left[S_{n}<0\right]>0$ for every $n \geq 1$. We are interested in the rate of decay of $\mathbb{P}\left[T_{0}>n\right]$ to zero, and we will see that far much less is known than for random walks.

The case of integrated simple random walks was first considered by Sinai [82], who showed the following

Theorem 3.1 (Sinai). Suppose that $\mu\{+1\}=\mu\{-1\}=1 / 2$. Then

$$
\mathbb{P}\left[T_{0}>n\right] \asymp n^{-1 / 4} .
$$

The main idea lying behind this theorem is very simple. Let $\left\{\tau_{k}, k \geq 0\right\}$ be the a.s. infinite sequence of return times of $S_{n}$ to zero viz. $\tau_{0}=0$ and $\tau_{k}=\inf \{n>$ $\left.\tau_{k-1}, S_{n}=0\right\}$. On the one hand, the simplicity assumption entails that $\left\{A_{\tau_{n}}, n \geq 1\right\}$ is an integer-valued symmetric random walk, and that the a.s. identification

$$
\left\{A_{\tau_{1}} \leq 0, \ldots, A_{\tau_{n}} \leq 0\right\}=\left\{A_{i} \leq 0, \forall i=1 \ldots \tau_{n}\right\}
$$

holds. By Gnedenko's local limit theorem, one has $\mathbb{P}\left[A_{\tau_{n}}=0\right] \sim k n^{-1 / 2}$ for some $k>$ 0 , so that the series $\sum_{n \geq 1} n^{-1} \mathbb{P}\left[A_{\tau_{n}}=0\right]$ is convergent. Sparre-Andersen's formula combined with the Tauberian theorem for monotonic sequences shows then that

$$
\mathbb{P}\left[T_{0}>\tau_{n}\right] \sim \frac{c}{\sqrt{n}} .
$$

On the other hand, the sequence $\tau_{n}$ grows like $n^{2}$ at infinity (more precisely, $n^{-2} \tau_{n}$ converges in law to some positive $(1 / 2)$-stable law), so that after some residual analysis on the bivariate random walk $\left\{A_{\tau_{n}}, \tau_{n}\right\}$, one obtains the desired result. Let us stress that the difficulty of the analysis stems from the fact that $\left(\tau_{n}\right)$ and $\left(A_{\tau_{n}}\right)$ are not independent. 
Since then several authors $([5,24,29,34,85,86])$ have tried to extend the validity of the estimate (3.1) to more general random walks. The following general result was proved very recently in [29], solving conjectures made in [24] and [84]:

Theorem 3.2 (Dembo-Ding-Gao). Suppose that $\mu$ has finite second moment and zero mean. Then for any $x \in \mathbb{R}$

$$
\mathbb{P}\left[T_{x}>n\right] \asymp n^{-1 / 4} .
$$

The method used by [29] is completely different from Sinai's and relies on a decomposition of the integrated walk at its supremum, which is somehow reminiscent of Sparre Andersen's argument, and too involved to be discussed here in detail. This method also allows for an elementary proof of the formula (2.5) in the symmetric and absolutely continuous case - see Proposition 1.4 therein.

The result in Theorem 3.2 was further refined in [34].

Theorem 3.3 (Denisov-Wachtel). Suppose that $\mu$ has finite $(2+\delta)$ moment for some $\delta>0$ and zero mean. Then there is a constant $c=c(\mu)>0$ such that for $x \in \mathbb{R}$

$$
\mathbb{P}\left[T_{x}>n\right] \sim c n^{-1 / 4} .
$$

Denisov and Wachtel use a new approach, based on their earlier results for other problems in [32]. They show that a typical scenario for $T_{0}>n$ is that $A$ becomes quite far negative over a short time period of order $n^{1-\varepsilon}$. From there, the pair $\left(S_{n}, A_{n}\right)$ essentially looks like a rescaled pair of Brownian motion and integrated Brownian motion $\left(B_{t}, \int_{0}^{t} B_{s} d s\right)$, which is formally obtained by coupling techniques similar to the idea applied in [5]. For the remaining time, $n-n^{1-\varepsilon} \sim n$, one requires the second component of the rescaled pair $\left(B_{t}, \int_{0}^{t} B_{s} d s\right)$ to be negative, which can be precisely analyzed using [47]. The paper [34] is also the first to apply potential-theoretic tools for persistence probabilities outside the classical random walk and Lévy process setting.

Sinai's method of decomposing the integrated path along the excursions away from zero of the underlying random walk can be extended under some extra assumptions on the law of $-X_{1}$ conditioned to be positive, which we denote by $\mu^{-}$. This idea had been used in [85] to show the same result as [29] in several situations like double-sided exponential, double-sided geometric, left-continuous, or lazy simple random walks. Recently in [86], this technique is combined with local limit theorems to obtain the following more precise result.

Theorem 3.4 (Vysotsky). Suppose that either $\mu_{-}$is exponential or that $\mu$ is leftcontinuous. If $\mu$ has finite variance and zero mean then there exists $c=c(\mu)>0$ such that 


$$
\mathbb{P}\left[T_{0}>n\right] \sim c n^{-1 / 4}
$$

Notice that there is still a small gap in that the existence of the constant in (3.2) is only shown under the conditions in Theorems 3.3 and 3.4, while in view of these results one would certainly expect it to hold under the sole assumption of finite variance. Even more, as can be seen from the central limit theorem, random walks with zero mean and finite variance are such that $\mathbb{P}\left[S_{n}>0\right] \rightarrow 1 / 2$. In view of the discussion made in Paragraph 2.1, it is hence natural to raise the more general

Conjecture 1 Suppose that $\mathbb{P}\left[S_{n}>0\right] \rightarrow 1 / 2$. Then

$$
\mathbb{P}\left[T_{0}>n\right]=n^{-1 / 4+o(1)} .
$$

In this formulation, no assumption is made on the moments of $\mu$ and this enhances sharply the difficulty of the problem, which probably requires more combinatorial tools than the one used in the above references.

We now turn to some situations where the persistence exponent of integrated random walks is not $1 / 4$. We denote by $\mathscr{D}(\alpha)$ the set of probability measures attracted to some strictly $\alpha$-stable law with $\alpha \neq 2$ and we refer e.g. to Chapter XVII.5 in [43] for more on the subject. Recall that if $\mu \in \mathscr{D}(\alpha)$, then $\mathbb{E}\left[\left|X_{1}\right|^{s}\right]<\infty$ for every $s \in[0, \alpha)$ and that $\mathbb{E}\left[X_{1}\right]=0$ if $\alpha>1$. The following is a consequence of the main result in [29]:

Theorem 3.5 (Dembo-Ding-Gao). Suppose that $\mu \in \mathscr{D}(\alpha)$ for some $\alpha \in(1,2)$. Then there exists an explicit constant $K$ such that

$$
\mathbb{P}\left[T_{0}>n\right] \leq K n^{-(1-1 / \alpha) / 2}, \quad n \geq 1
$$

If in addition $\mu$ is attracted to a spectrally positive $\alpha$-stable law, then

$$
\mathbb{P}\left[T_{0}>n\right] \asymp n^{-(1-1 / \alpha) / 2} .
$$

The result in [29] is formulated in a different manner and is actually more general. In the case when $\mu \in \mathscr{D}(\alpha)$ however, the additional assumption made therein for the lower bound is equivalent to the spectral positivity of the attracting law - see e.g. Theorem XVII.5.1 in [43]. With the help of an extension of Sinai's method, the estimate was recently refined in [86]:

Theorem 3.6 (Vysotsky). Suppose that $\mu_{-}$is exponential or that $\mu$ is left-continuous. If $\mu$ is centered, attracted to some spectrally positive $\alpha$-stable law with $\alpha \in(1,2)$, and if

$$
\sum_{n \geq 1} \frac{1}{n}\left(\mathbb{P}\left[S_{n}<0\right]-\frac{1}{\alpha}\right)<+\infty,
$$

then there exists $c=c(\mu)>0$ such that 


$$
\mathbb{P}\left[T_{0}>n\right] \sim c n^{-(1-1 / \alpha) / 2} .
$$

In view of our final discussion made in Paragraph 2.1, it is very surprising that the case where $\mu$ is right-continuous and normally attracted to some spectrally negative $\alpha$-stable law seems to be more difficult to handle than the dual situation where $\mu$ is left-continuous and normally attracted to some spectrally positive $\alpha$-stable law.

It is natural to ask what the persistence exponent should be when the limit stable law does have negative jumps. Let us hence denote by $\mathscr{D}(\alpha, \rho)$ the set of probability measures attracted to a strictly $\alpha$-stable law with positivity parameter $\rho$. Notice - see e.g. [89] for details and recall that we excluded the one-sided case - that $\rho \in(0,1)$ for $\alpha \in(0,1]$, that $\rho \in[1-1 / \alpha, 1 / \alpha]$ for $\alpha \in(1,2)$, and that spectrally positive $\alpha$-stable laws with $\alpha \in(1,2)$ are such that $\rho=1-1 / \alpha$. Besides, one has $\mathbb{P}\left[S_{n}>0\right] \rightarrow \rho$ whenever $\mu \in \mathscr{D}(\alpha, \rho)$ - see e.g. Theorem XVII.5.1 in [43]. In view of the discussion made in Paragraph 2.1, it is natural to raise the general

Conjecture 2 Suppose that $\mathbb{P}\left[S_{n}>0\right] \rightarrow \rho \in(0,1)$. Then

$$
\mathbb{P}\left[T_{0}>n\right]=n^{-\rho / 2+o(1)} .
$$

A weak version of this conjecture is to get the asymptotic under the assumption that $\mu \in \mathscr{D}(\alpha, \rho)$. Under the stronger assumption that $\mu$ is normally attracted, one may also wonder if a more precise behavior could not be obtained, as in [86].

\subsection{Integrated Lévy processes}

In this section we consider the process

$$
A_{t}=\int_{0}^{t} Z_{s} \mathrm{~d} s, \quad t \geq 0,
$$

where $\left\{Z_{t}, t \geq 0\right\}$ is a real Lévy process starting from zero. We set

$$
T_{x}=\inf \left\{t>0, A_{t}>x\right\}=\inf \left\{t>0, A_{t}=x\right\}
$$

for its first passage time above $x \geq 0$. Contrary to (2.11), there is no straightforward bound between $\mathbb{P}\left[T_{x} \geq t\right]$ and an analogous quantity involving some iterated random walk, so that the results of the previous paragraph cannot be used, except in the Brownian case, see [29] Remark 1.5.

The process $(Z, A)$ is Fellerian and we set $\mathbb{P}_{(z, a)}$ for its law starting from $(z, a)$, with the simplified notation $\mathbb{P}=\mathbb{P}_{(0,0)}$. It is clear by the right-continuity of $Z$ that

$$
\mathbb{P}_{(z, 0)}\left[T_{0}=0\right]=1 \text { or } 0 \quad \text { according as } z>0 \text { or } z<0 .
$$


Since $T_{0}$ is the first passage time into the positive half-space for the Fellerian process $(Z, A)$, one has also $\mathbb{P}\left[T_{0}=0\right]=0$ or 1 by the $0-1$ law, but to obtain a criterion for the regularity of the upper half-plane for $(Z, A)$ is an open problem which does not seem obvious. Since integrated Lévy process all have finite variation, one might wonder whether this criterion would not be different from the aforementioned Theorem 22 in [39].

If $Z$ drifts to $+\infty$, then it is clear that $T_{x}<+\infty$ a.s. for every $x>0$. On the other hand, if $Z$ drifts to $-\infty$ then its last passage time above zero can be made arbitrarily small so that one will have $\mathbb{P}\left[T_{x}=+\infty\right]>0$ for every $x>0$. When $Z$ oscillates then probably one has $\mathbb{P}\left[T_{x}=+\infty\right]=0$ for every $x>0$, but there is no direct answer to this question. In general there is no result of basic fluctuation theory available for integrated Lévy processes.

In this paragraph we will consider two examples where the persistence exponent can be computed. The first one is the integrated Brownian motion and originates from Kolmogoroff [56], in relation with the two-dimensional generator

$$
\frac{1}{2} \frac{\partial^{2}}{\partial x^{2}}+x \frac{\partial}{\partial y}
$$

and the associated Fokker-Planck equation. Notice that [56] actually deals with the more general $n$-times integrated Brownian motion. The process $(B, A)$ is a Gaussian Markov process whose transition density can be computed explicitly via the covariance matrix. By the (3/2)-self-similarity of $A$, one has

$$
T_{x} \stackrel{d}{=} x^{2 / 3} T_{1}
$$

under $\mathbb{P}$, so that our persistence problem amounts to find the asymptotic of $\mathbb{P}\left[T_{1}>t\right]$, a question which dates back to Uhlenbeck and Wang in 1945. Notice that the above identity also yields $\mathbb{P}\left[T_{0}=0\right]=1$. Among other formulæ, the following was obtained by McKean in an analytical way - see (3.1) in [65]:

$$
\begin{aligned}
& \mathbb{P}_{(0,-1)}\left[T_{0} \in \mathrm{d} t, B_{T_{0}} \in \mathrm{d} x\right]=\frac{3 x}{\pi \sqrt{2 \pi} t^{2}} e^{-(2 / t)\left(1-x+x^{2}\right)}\left(\int_{0}^{4 x / t} e^{-3 y / 2} y^{-1 / 2} \mathrm{~d} y\right) \\
& \mathbf{1}_{\{x \geq 0\}} \mathrm{d} t \mathrm{~d} x .
\end{aligned}
$$

This formula is the key argument to the following result which is proved separately in [46] and [51]:

Theorem 3.7 (Goldman, Isozaki-Watanabe). With the above notations there exists ac $>0$ such that

$$
\mathbb{P}\left[T_{1}>t\right] \sim c t^{-1 / 4} .
$$

Notice that by self-similarity this result has the more general formulation

$$
\mathbb{P}\left[A_{s} \leq x, \forall s \in[0, t]\right]=\mathbb{P}\left[T_{x}>t\right] \sim c x^{1 / 6} t^{-1 / 4} \quad \text { as } t / x^{2 / 3} \rightarrow+\infty .
$$


In particular, one has

$$
\mathbb{P}\left[A_{s} \leq x, \forall s \in[0,1]\right] \sim c x^{1 / 6} \quad \text { as } x \rightarrow 0,
$$

which is a lower tail probability statement as mentioned in the introduction. By approximation and his result on integrated simple random walks, Sinai had obtained in [82] the rougher estimate

$$
\mathbb{P}\left[T_{1}>t\right] \asymp t^{-1 / 4} .
$$

The argument in [46] is analytical and relies on integral equations. It also yields a complicated explicit expression for the law of $T_{1}$ :

$$
\begin{aligned}
\mathbb{P}\left[T_{1} \in \mathrm{d} t\right]= & \mathbb{P}_{(0,-1)}\left[T_{0} \in \mathrm{d} t\right]=\left(\frac{3 \sqrt{3}}{2 \sqrt{2 \pi} t^{5 / 2}} e^{-3 /\left(2 t^{3}\right)}\right. \\
+ & \frac{2 \sqrt{3}}{\pi} \int_{0}^{\infty} \int_{0}^{\infty} \int_{0}^{t} \mathbb{P}_{(-z, 0)}\left[t-T_{0} \in d s, B_{T_{0}} \in \mathrm{d} x\right] \\
& \left.\cdot e^{-6 / s^{3}-2 z^{2} / s^{2}} \sinh \left(6 z / s^{2}\right) z d z d s\right) \mathrm{d} t
\end{aligned}
$$

where the quantity $\mathbb{P}_{(-z, 0)}\left[t-T_{0} \in d s, B_{T_{0}} \in \mathrm{d} x\right]$ can be expressed via McKean's above formula and self-similarity. Notice that both McKean and Goldman's formulæ have been generalized by Lachal [59], who obtained an explicit formula for $\mathbb{P}_{(b, a)}\left[T_{0} \in \mathrm{d} t, B_{T_{0}} \in \mathrm{d} x\right]$ for any $(b, a)$. The asymptotic analysis which is carried out in Proposition 2 of [46] gives the right speed of convergence for $\mathbb{P}[T \in \mathrm{d} t]$, but the resulting value of the constant $c$ in (3.3) is erroneous because of some inaccuracies in the change of variable. The right value is

$$
c=\frac{3^{4 / 3} \Gamma(2 / 3)}{\pi 2^{13 / 12} \Gamma(3 / 4)} \sim 0.718
$$

and follows also from the simpler probabilistic method of [51], which relies on the Markov property and a Tauberian argument. This method yields the more general estimate

$$
\mathbb{P}_{(b, 0)}\left[T_{x}>t\right] \sim c_{b, x} t^{-1 / 4}
$$

with some explicit $c_{b, x}>0$ - see (1.12) in [51]. It also allows to handle first-passage time asymptotics for fluctuating homogeneous additive functionals of Brownian motion, with the persistence exponent depending smoothly on the skewness of the functional - see Corollary 1 in [50]. We finally notice that the estimate (3.3) was also obtained (with an erroneous constant $c$ ) in [23] after solving some Krein-Kramers differential equation in the context of semiflexible polymers in the half-plane. This latter method was generalised in [20] to give another computation of the persistence exponent for fluctuating homogeneous additive functionals of Brownian motion, in the context of survival of a diffusing particle in a transverse shear flow. Let us also mention the paper [47], which obtains precise informations on the law of integrated 
Brownian motion conditioned to stay positive.

We now turn to integrated strictly $\alpha$-stable Lévy processes, which form the natural generalisation of integrated Brownian motion. The bivariate process $(Z, A)$ is then a stable Markov process, where the stability property has the general meaning which is given in the monograph [76]. In particular, one can check from the Lévy-Khintchine formula - see e.g. Proposition 3.4.1 in [76] - that

$$
A_{1} \stackrel{d}{=}(1+\alpha)^{-1 / \alpha} Z_{1}
$$

for every $t \geq 0$, so that $A_{1}$ is a $\alpha-$ stable variable with the same positivity parameter as $Z_{1}$. On the other hand there does not exist an explicit formula for the density of the bivariate random variable $\left(Z_{1}, A_{1}\right)$ except in the Gaussian case $\alpha=2$. The univariate process $A$ is $(1+1 / \alpha)$-self-similar, so that with the above notations one has

$$
T_{x} \stackrel{d}{=} x^{\alpha /(\alpha+1)} T_{1}
$$

under $\mathbb{P}$ and we need only to study the asymptotic behavior of $\mathbb{P}\left[T_{1}>t\right]$. Notice that again this identity yields $\mathbb{P}\left[T_{0}=0\right]=1$. As a consequence of the main result of [80], the persistence exponent of $A$ can be computed within a specific sub-class:

Theorem 3.8 (Simon). Let $\left\{Z_{t}, t \geq 0\right\}$ be a strictly $\alpha$-stable Lévy process with $\alpha \in$ $(1,2)$. With the above notations, there exists a positive constant $K$ such that

$$
\mathbb{P}\left[T_{1}>t\right] \leq K t^{-(1-1 / \alpha) / 2}, \quad t>0
$$

If in addition $Z$ is spectrally positive, then

$$
\mathbb{P}\left[T_{1}>t\right]=t^{-(1-1 / \alpha) / 2+o(1)} .
$$

The main result in [80] deals with more general homogeneous functionals of stable Lévy processes, extending the results of [49]. It also provides some explicit lower bound with a logarithmic term, which entails the following criterion for the finiteness of fractional moments of $T_{1}$ in the spectrally positive case:

$$
\mathbb{E}\left[T_{1}^{S}\right]<\infty \Leftrightarrow-(\alpha+1)<s<(\alpha-1) / 2 \alpha \text {. }
$$

The method of [80] is an adaptation of Sinai's in continuous time, relying on the bivariate Lévy process $\left\{\left(\tau_{t}, A_{\tau_{t}}\right), t \geq 0\right\}$ with $\left\{\tau_{t}, t \geq 0\right\}$ the inverse local time at zero (which exists because $\alpha>1$ ). The upper bound relies on the Wiener-Hopf factorization method as in [49], and the fact that $A_{\tau_{1}}$ is a symmetric $(\alpha-1) /(\alpha+$ 1)-stable variable whatever $A_{1}$ 's positivity parameter is. For the lower bound, the crucial fact is that a.s.

$$
\left\{A_{\tau_{s}} \leq 1, \forall s \in[0, t]\right\}=\left\{A_{s} \leq 1, \forall s \in\left[0, \tau_{t}\right]\right\}
$$


which allows to study the probability of the right event with the help of (2.17). This latter identity is true only in the spectrally positive case.

It is a natural question to find the persistence exponent for all integrated stable Lévy processes. If $Z$ is an $\alpha$-stable subordinator, then $A$ is an increasing process and one gets the same superexponential behavior as in (2.2). This superexponential speed is coherent with the previous discussion for integrated one-sided random walks. If $-Z$ is an $\alpha$-stable subordinator, then $T_{x}=+\infty$ a.s. for every $x \geq 0$. The general problem remains open, and in view of Bingham's estimate (2.17) it is natural to raise the precise

Conjecture 3 Let $Z$ be a strictly $\alpha$-stable Lévy process such that $\mathbb{P}\left[Z_{1}>0\right]=\rho \in$ $(0,1)$. Then there exists $c>0$ such that

$$
\mathbb{P}\left[T_{1}>t\right] \sim c t^{-\rho / 2} .
$$

We see that the main result of [80] shows the conjecture in the spectrally positive case viz. $\alpha>1$ and $\rho=1-1 / \alpha$, except the existence of the constant for which the refined methods of [86] could perhaps be adapted. In the general case, a simple scenario with an initial downward intention is described in [36] p. 3 to show that the speed must be larger than $t^{-} \rho$. Unfortunately, the attempts made therein to obtain a better bound were unjustified and in [81] a method relying on decorrelation inequalities, inspired by [24], is carried out to show that the upper bound on the probability in the main result of [80] is not the optimal one when $Z$ has negative jumps and self-similarity index close to 1 :

Theorem 3.9 (Simon). For every $\alpha_{0}, c>0$ there exists $k>0$ such that for every $\alpha \in\left[\alpha_{0}, 2\right]$ and every strictly $\alpha$-stable process $Z$ with positivity parameter $\rho \in$ $[c \wedge(1-1 / \alpha), 1 / \alpha \vee 1]$ one has

$$
\liminf _{t \rightarrow \infty} t^{k} \mathbb{P}\left[T_{1}>t\right]=0 .
$$

At present, it does not seem that the different arguments developed in [36, 80, 81] can provide any clue to get the conjectured persistence exponent $\rho / 2$. It is quite surprising that when $Z$ is spectrally positive the persistence exponent is easier to get for $A$ than for $-A$. Indeed, the simple identity (2.18) entails readily that the persistence exponent of $-Z$ is $1 / \alpha$, whereas it is not a straightforward task to show that the persistence exponent of $Z$ is $1-1 / \alpha$.

It seems plausible that a version without constant of Conjecture 3 could be proved in refining the results of [29] and using some discrete approximation. In view of Conjecture 2 and (2.15), this leads us to the following general question on integrated Lévy processes:

Conjecture 4 Let $Z$ be a Lévy process such that $\mathbb{P}\left[Z_{t}>0\right] \rightarrow \rho \in(0,1)$ as $t \rightarrow+\infty$. Then for every $x>0$

$$
\mathbb{P}\left[T_{x}>t\right]=t^{-\rho / 2+o(1)} .
$$




\subsection{Fractionally integrated Lévy processes}

In this section we consider processes of the type

$$
A_{t}^{\beta}=\frac{1}{\Gamma(\beta+1)} \int_{0}^{t}(t-s)^{\beta} \mathrm{d} Z_{s}, \quad t \geq 0,
$$

where $\left\{Z_{t}, t \geq 0\right\}$ is a real Lévy process starting from zero and $\beta>-1$. The above convolution product makes sense for every $\beta \geq 0$, and can also be defined for some negative $\beta$ depending on the law of $Z$. If $Z$ is strictly $\alpha$-stable for instance, then $A^{\beta}$ is well-defined for every $\beta>-(1 \wedge 1 / \alpha)$ and is then a stable process in the sense of [76], with continuous paths iff $\alpha=2$ or $\beta>0$ and with a.s. locally unbounded paths iff $\alpha<2$ and $\beta<0$ - see Chapter 10 in [76]. When $Z$ is strictly $\alpha$-stable, it is customary to write $\beta=H-1 / \alpha$ with $H>0$ the so-called Hurst parameter, and $A^{\beta}$ is then an $H$-self-similar process. One can view $\beta$-fractionally integrated Lévy processes as the natural generalization of $n$ times integrated Lévy processes, since an integration by parts shows that the latter form the subclass $\beta=n$. In this particular case the $(n+1)$-dimensional process $\left(Z, A^{1}, \ldots, A^{n}\right)$ is a strong Markov process, but there is no multidimensional Markov property when $\beta$ is not an integer because then the fractional integration takes the whole memory of the driving process into account. In the literature fractionally integrated Lévy processes are often called Riemann-Liouville processes, a denomination which is originally due to Lévy.

In the Brownian case $Z=B$, the process $A^{\beta}$ is closely connected to the fractional Brownian motion $\left\{B_{t}^{H}, t \geq 0\right\}$, which we recall to be the centered Gaussian process with covariance function

$$
\mathbb{E}\left[B_{t}^{H} B_{s}^{H}\right]=\frac{1}{2}\left(t^{2 H}+s^{2 H}-|t-s|^{2 H}\right), \quad t, s \geq 0 .
$$

Fractional Brownian motion can be written as the independent sum

$$
B_{t}^{H}=c_{H}\left(A_{t}^{H-1 / 2}+\int_{0}^{\infty}\left((t+s)^{H-1 / 2}-s^{H-1 / 2}\right) \mathrm{d} \tilde{B}_{s}\right), \quad t \geq 0
$$

with $c_{H}=\left(H 2^{2 H} \pi /(\Gamma(H+1 / 2) \Gamma(1-H))\right)^{1 / 2}$ the normalization constant and $\tilde{B}$ a Brownian motion independent of $B$, which shows that its paths are continuous a.s. Notice that $B^{H}$ gives insight on the process of the long-range increments of $A^{H-1 / 2}$ in view of the immediate representation of the latter as an independent sum

$$
A_{t+u}^{H-1 / 2}-A_{u}^{H-1 / 2} \stackrel{d}{=} A_{t}^{H-1 / 2}+\int_{0}^{u}\left((t+s)^{H-1 / 2}-s^{H-1 / 2}\right) \mathrm{d} \tilde{B}_{s}, \quad t \geq 0,
$$


which entails

$$
\left\{A_{t+u}^{H-1 / 2}-A_{u}^{H-1 / 2}, t \geq 0\right\} \stackrel{d}{\rightarrow}\left\{c_{H}^{-1} B_{t}^{H}, t \geq 0\right\}, \quad u \rightarrow+\infty
$$

(with, of course, an equality in law for every $u$ when $H=1 / 2$ ). Recall that $B^{H}$ is defined for $H \in(0,1]$ only and that $B^{1}$ is simply the linear function $t \mapsto t N$ with $N$ a standard normal variable. Fractional Brownian motion can be shown [76] to be the unique $H$-self-similar Gaussian process with stationary increments, whence its greater importance in modeling than fractionally integrated Lévy processes. Setting

$$
T_{x}^{H}=\inf \left\{t>0, B_{t}^{H}>x\right\}=\inf \left\{t>0, B_{t}^{H}=x\right\}
$$

for every $x \geq 0$, one has $T_{x}^{H} \stackrel{d}{=} x^{1 / H} T_{1}^{H}$ by self-similarity, which shows that $T_{0}^{H}=0$ a.s. and that the survival analysis of $B^{H}$ is reduced to the behavior of $\mathbb{P}\left[T_{1}^{H}>t\right]$ only. Among other results, the following was obtained in [67]:

Theorem 3.10 (Molchan). For every $H \in(0,1]$ one has

$$
\mathbb{P}\left[T_{1}^{H}>t\right]=t^{H-1+o(1)} .
$$

The main argument of [67], partly inspired by Brownian fluctuation theory, is to quantify the correlation between $T_{1}^{H}$, the last zero of $B^{H}$ on $[0,1]$, the positive sojourn time of $B^{H}$ on $[0,1]$, and the inverse exponential functional

$$
t \mapsto J_{t}^{H}=\left(\int_{0}^{t} e^{B_{s}^{H}} \mathrm{~d} s\right)^{-1},
$$

whose asymptotic behavior in expectation can be precisely analysed. The general link between $J_{t}^{H}$ and $T_{1}^{H}$ is explained by the heuristical fact that if $T_{1}^{H}>t$ then $B^{H}$ has drifted towards $-\infty$ rather rapidly, so that $J_{t}^{H}$ is big. Conversely if $T_{1}^{H}<t$ then $B^{H}$ has been close zero for a positive fraction of time, so that $J_{t}^{H}$ is small. However, the analysis of $\left\{\mathbb{E}\left[J_{t}^{H}\right], t \geq 0\right\}$ which is performed in [67] is very specific to the stationary increments of fractional Brownian motion, and does not seem to be suitable for fractionally integrated Lévy processes. However, it can indeed be used for other self-similar, stationarity increment processes with sufficiently high integrability properties, as for example done in [25], where the following process is considered:

$$
\Delta_{t}=\int_{\mathbb{R}} L_{t}(x) d W(x), \quad t \geq 0,
$$

with $W$ being a two-sided Brownian motion and $L_{t}$ the local time of an $\alpha$-stable Lévy process $(\alpha \in(1,2])$ independent of $W$. This process is $H=(1-1 /(2 \alpha))$-selfsimilar, has stationary increments, and can be analyzed with the techniques from $[2,67]$ to show that the persistence exponent is $1 /(2 \alpha)=1-H$. Similarly, [26] consider more general types of processes yielding the local time process $L$. We further mention [72, 4], where the persistence problem with moving boundary is studied, as well as moments of $J_{T}$ and probabilities of scenarios that are very similar to per- 
sistence.

Continuing our discussion on FBM, note that it is clear that $\mathbb{P}\left[T_{1}^{1}>t\right] \rightarrow 1 / 2$ by the above remark on the case $H=1$, and it is natural to raise the

Conjecture 5 For every $H \in(0,1)$ one has

$$
\mathbb{P}\left[T_{1}^{H}>t\right] \asymp t^{H-1} .
$$

In view of the previously stated precise results, one may also ask if $\mathbb{P}\left[T_{1}^{H}>t\right] \sim$ $c t^{H-1}$.

The above conjecture was recently addressed in [2], where the following partial result is obtained by a refinement of Molchan's method:

Theorem 3.11 (Aurzada). There exists $c>0$ such that

$$
(\log t)^{-c} t^{H-1} \leq \mathbb{P}\left[T_{1}^{H}>t\right] \leq(\log t)^{c} t^{H-1}, \quad t \rightarrow+\infty .
$$

We now turn to first passage asymptotics for a class of Gaussian stationary processes (GSP) which are related to the persistence of fractionally integrated Brownian motion. In [62] the Lamperti transformation

$$
L_{t}^{H}=e^{-t H} B_{e^{t}}^{H}, \quad t \in \mathbb{R},
$$

a centered GSP, is studied in connection with the persistence exponent of $B^{H}$ and it is shown that

$$
\log \mathbb{P}\left[L_{s}^{H} \leq 0, \forall s \in[0, t]\right]=\log \mathbb{P}\left[B_{s}^{H} \leq 0, \forall s \in\left[1, e^{t}\right]\right] \sim t(H-1) .
$$

Since Molchan's theorem means that $\log \mathbb{P}\left[B_{s}^{H} \leq 1, \forall s \in\left[0, e^{t}\right]\right] \sim t(H-1)$, one simply needs to switch the 0 and the 1 in the latter probability to obtain this result, which is justified in [62] through a refined use of Slepian's lemma. The Lamperti transformation is also a fruitful method in the reverse direction, and this was observed in [5] to investigate the persistence exponents of fractionally integrated Lévy processes. Assume that $Z$ in (3.4) is a Brownian motion and introduce the notation

$$
T_{x}^{\beta}=\inf \left\{t>0, A_{t}^{\beta}>x\right\}=\inf \left\{t>0, A_{t}^{\beta}=x\right\}
$$

for every $\beta, x \geq 0$. Notice that $T_{x}^{\beta} \stackrel{d}{=} x^{2 /(1+2 \beta)} T_{1}^{\beta}$ by self-similarity, which shows that $T_{0}^{\beta}=0$ a.s. and that the survival analysis of $B^{H}$ is reduced to the behavior of $\mathbb{P}\left[T_{1}^{\beta}>t\right]$ only. The process

$$
Y_{t}^{H}=e^{-t H} A_{e^{t}}^{H-1 / 2}, \quad t \in \mathbb{R},
$$

a centered GSP for every $H>0$ which reduces to the stationary Ornstein-Uhlenbeck process when $H=1 / 2$, plays a key rôle in the following theorem ([5]). 
Theorem 3.12 (Aurzada-Dereich). There exists a non-increasing function $\beta \mapsto$ $\theta(\beta)$ such that

$$
\mathbb{P}\left[T_{1}^{\beta}>t\right]=t^{-\theta(\beta)+o(1)}
$$

for every $\beta \geq 0$. Besides one has $\theta(\infty)=-\lim _{t \rightarrow \infty} t^{-1} \log \mathbb{P}\left[Y_{s}<0, \forall s \in[0, t]\right]$, where $Y$ is the centered GSP with correlation $\mathbb{E}\left[Y_{0} Y_{t}\right]=1 / \cosh (t / 2)$.

The important process $Y$ is mentioned in [63] in the context of diffusion equation with white noise initial conditions, and in [31] in relation with the positivity of random polynomials with large even degree. More details will be given in the next section. In [62], the process $Y$ is also viewed as the Lamperti transformation of the curious smooth (1/2)-self-similar Gaussian process

$$
X_{t}=\sqrt{2} t^{2} \int_{0}^{\infty} B_{u} e^{-u t} d u, \quad t \geq 0,
$$

which shares the same time inversion property $\left\{X_{t}, t>0\right\} \stackrel{d}{=}\left\{t^{-1} X_{t^{-1}}, t>0\right\}$ as Brownian motion. In [5] only the upper bound

$$
-\lim _{t \rightarrow \infty} t^{-1} \log \mathbb{P}\left[Y_{s}<0, \forall s \in[0, t]\right] \leq \theta(\infty)
$$

is proved via Slepian's lemma, but one can easily show that $Y^{H} \stackrel{d}{\rightarrow} Y$ as $H \rightarrow \infty$ in analysing the covariance function, so that the inequality in (3.9) is actually an equality, the limit on the left-hand side being a supremum. This also follows from the more general Theorem 1.6 in [30].

Theorem 3.12 has several interesting consequences. First, it shows that the persistence exponent is a non-increasing function of the order of integration for Brownian fractionally integrated processes. The fact that smoother processes have more probability to survive is believed to be a kind of universal feature. Second, it entails that $\theta(\beta) \geq \theta(1)=1 / 4$ for every $\beta \leq 1$, so that by Molchan's result the persistence exponents of $B^{H}$ and $A^{H-1 / 2}$ do not coincide whenever $H>3 / 4$. We actually believe that $\theta(H-1 / 2)>1-H$ for every $H \in(1 / 2,1)$ and some reasons for that will be given soon afterwards. Last, it entails that $\theta(\infty) \leq \theta(1)=0.25$, which improves the bound $\theta(\infty)<0.325$ obtained in [62] via another Slepian's inequality. In [63] the numerical value $\theta(\infty) \sim 0.1875$ is suggested, whereas in [31] the value $\theta(\infty)=0.19 \pm 0.01$ is obtained by simulations. It is a tantalizing question to compute the function $\theta(\beta)$ for every positive $\beta \notin\{0,1\}$, as well as its limit $\theta(\infty)$. The lower bound $\theta(\infty)>0.125$ is obtained in [61] with the help of a certain Gaussian comparison inequality, and in [69] this lower bound is improved into $\theta(\infty)>1 /(4 \sqrt{3})>0.144$, in comparing $Y$ with a linear time-change of the so-called Wong process [88], which is the GSP associated with integrated Brownian motion.

Let us give some more details on the correlation function $C_{H}(t)=\mathbb{E}\left[Y_{0}^{H} Y_{t}^{H}\right]$ of the Lamperti transform introduced in (3.8). It is given by 


$$
C_{H}(t)=2 e^{-H t} \int_{0}^{1}\left(u\left(u+e^{t}-1\right)\right)^{H-1 / 2} \mathrm{~d} u
$$

for every $t \geq 0$, and a simple analysis shows that for every $H \in(0,1]$ and $t \geq 0$ one has

$$
C_{H}(t) \leq \cosh (H t)-2^{2 H-1}(\sinh (t / 2))^{2 H}=\mathbb{E}\left[L_{0}^{H} L_{t}^{H}\right],
$$

where $L^{H}$ is the Lamperti transform defined in (3.7). By Slepian's lemma and Molchan's theorem, this entails

$$
\theta(H-1 / 2) \geq 1-H
$$

for every $H \in[1 / 2,1]$, with an equality if $H=1 / 2$. From the above we know that (3.10) is strict if $H>3 / 4$. It is very likely that $\theta(H-1 / 2)$ exists for every $H \in$ $(0,1 / 2)$ and that (3.10) is strict for every $H \neq 1 / 2$. In view of the above discussion, it is natural to raise the

Conjecture 6 The function $\beta \mapsto \theta(\beta)$ is convex decreasing.

We now go back to general fractionally integrated Lévy processes and state the following result of [5], which is obtained by strong approximation and drift transformations of Gaussian processes:

Theorem 3.13 (Aurzada-Dereich). For every $\beta \geq 0$, the persistence exponent $\theta(\beta)$ is the same among all $\beta$-fractionally integrated centered Lévy processes such that $t \mapsto \mathbb{E}\left[e^{t Z_{1}}\right]$ is finite in an open neighborhood of zero.

The main result of [5], to which we refer for details, is more general and handles fractionally integrated random walks as well as more general Volterra kernels. The finiteness of exponential moments play an important rôle therein due to the use of strong approximation, but in view of the aforementioned results and conjectures on integrated random walks and Lévy processes, it is natural to raise the

Conjecture 7 For every $\beta \geq 0$, the exponent $\theta(\beta)$ is the same among all $\beta$-fractionally integrated centered Lévy processes with finite variance.

We remark that there are many other types of fractional Lévy processes than fractionally integrated ones - see e.g. [41] for a recent account, but up to the knowledge of the authors no results for their persistence probabilities are known.

Let us conclude this paragraph with the integrated fractional Brownian motion

$$
I_{t}^{H}=\int_{0}^{t} B_{s}^{H} d s, \quad t \geq 0 .
$$

This is a centered Gaussian process with positive correlation function, and with the help of its Lamperti transformation it is shown easily using Slepian's inequality, subadditivity, and e.g. Proposition 1.6 in [5] that for every $H \in(0,1)$ there exists $\rho(H)>0$ such that 


$$
\mathbb{P}\left[I_{s}^{H} \leq 1, \forall s \in[0, t]\right]=t^{-\rho(H)+o(1)} .
$$

Numerical simulations [70] suggest the following:

Conjecture 8 (Khokhlov-Molchan) One has $\rho(H)=H(1-H)$.

This expected value, which is symmetric with respect to $H=1 / 2$, is very surprising because it is known that fractional Brownian motions with Hurst index smaller or greater than $1 / 2$ are very different processes from several viewpoints. This does not match either the above heuristic discussion on smooth GSP's since the correlation function $F^{H}$ of the Lamperti transform of $I^{H}$ has a first-order expansion at zero

$$
F^{H}(t)=1-\frac{\left(1-H^{2}\right) t^{2}}{2}+o\left(t^{2}\right)
$$

In particular, the number $N_{t}^{H}$ of zero-crossings of this Lamperti process has an expectation $\mathbb{E}\left[N_{t}^{H}\right]=t \sqrt{1-H^{2}} / \pi$ which decreases with $H$, and one might think that $\rho(H)$ also decreases. However integrated fractional Brownian motion may well be more the exception than the rule for this kind of questions, because of its complicated correlation structure. In [70] it is argued that the difference between $H<1 / 2$ and $H>1 / 2$ should be observed at the logarithmic level and it is shown in [68], with a detailed analysis, that $c H(1-H) \leq \rho(H) \leq 1-H$ for some $c \in(0,1)$. Other bounds such as $\rho(H) \geq \rho(1-H)$ for every $H \leq 1 / 2$ were also recently presented in [69] (with numerical explanations), neither proving nor disproving the above conjecture.

\subsection{Other processes}

Integrated random walks which were considered previously can be written as the weighted sum

$$
A_{n}=\sum_{i=1}^{n}(n+1-i) X_{i}
$$

where $\left\{X_{i}, i \geq 1\right\}$ is an i.i.d. sequence. The above weights depend on $i$ and $n$ so that the increments of $A_{n}$ are neither stationary nor independent. It is natural to consider persistence problem for other weighted sums like weighted random walks:

$$
\Sigma_{n}=\sum_{i=1}^{n} \sigma_{i} X_{i}
$$

where $\left\{\sigma_{i}, i \geq 1\right\}$ is a deterministic sequence and $\left\{X_{i}, i \geq 1\right\}$ an i.i.d. family. The situation is a bit simpler than for integrated random walks because the non-stationary increments of $\Sigma$ are independent, nevertheless the sequence $\left\{\sigma_{i}, i \geq 1\right\}$ has also more generality. Setting $\mu$ for the law of $X_{1}$ and introducing

$$
T_{\sigma}=\inf \left\{n \geq 1, \Sigma_{n}>0\right\},
$$


the following has been obtained in [3] via strong approximation techniques:

Theorem 3.14 (Aurzada-Baumgarten). Suppose that $\mu$ is centered and that its Laplace transform is finite in an open neighborhood of zero. Suppose that $\left\{\sigma_{i}, i \geq 1\right\}$ is increasing with $\sigma_{n} \asymp n^{p}$ for some $p>0$. Then

$$
\mathbb{P}\left[T_{\sigma}>n\right]=n^{-(p+1 / 2)+o(1)} .
$$

The results of [3] are more precise and allow other weight functions not necessarily increasing when $\mu$ is Gaussian. The general case reduces to the Gaussian one via strong approximation and drift transformations, as in [5]. A universal speed not depending on $\mu$ can also be obtained for weight functions growing faster than polynomials, like $e^{n^{\gamma}}$ for some $\gamma<1 / 4$, and the persistence probability then has streched exponential decay. However, it is also shown in [3] that the speed does depend on $\mu$ for weight functions growing too fast, like $e^{n^{\gamma}}$ for some $\gamma \geq 1$. It would be interesting to find $\sigma$ 's critical growth rate for the universality of the speed. The following is also a natural question.

Conjecture 9 Suppose that $\mu$ is centered and has finite variance. Suppose that $\left\{\sigma_{i}, i \geq 1\right\}$ is increasing with $\sigma_{n} \asymp n^{p}$ for some $p>0$. Then

$$
\mathbb{P}\left[T_{\sigma}>n\right]=n^{-(p+1 / 2)+o(1)} .
$$

Let us now consider iterated Lévy processes, which are processes of the type $\left\{X \circ\left|Y_{t}\right|, t \geq 0\right\}$ with $X, Y$ two independent real Lévy processes starting from zero. If $|Y|$ is a subordinator, then $X \circ|Y|$ is another Lévy process which is called a subordinate Lévy process, a notion introduced by Bochner in the context of harmonic analysis. Iterated Lévy processes were introduced by Burdzy in the Brownian framework and can be viewed as a generalisation of subordinate Lévy processes. They are known to have strong connections with PDE's of higher order, especially through their first passage times. Let us introduce

$$
T_{x}=\inf \left\{t>0, X \circ\left|Y_{t}\right|>1\right\}
$$

for all $x \geq 0$, and notice that as for integrated Lévy processes the law of $T_{x}$ is difficult to study in general since $X \circ|Y|$ is non-Markov. Among other results, the following was obtained in [12] and subsequently improved by [87]:

Theorem 3.15 (Baumgarten, Vysotsky). Suppose that the random variables $X_{1}$ and $Y_{1}$ have finite variance and $\mathbb{E}\left[X_{1}\right]=0$. Then

$$
\begin{aligned}
& \mathbb{P}\left[T_{1}>t\right] \asymp t^{-1 / 4} \quad \text { for } \mathbb{E}\left[Y_{1}\right]=0, \\
& \mathbb{P}\left[T_{1}>t\right]=t^{-1 / 2+o(1)} \quad \text { for } \mathbb{E}\left[Y_{1}\right] \neq 0,
\end{aligned}
$$

where for the second statement one has to assume that $X_{1}$ and $Y_{1}$ have some finite stretched exponential moment. 
In particular, the persistence exponent of iterated Brownian motion is $1 / 4$. The strong dichotomy between the situations where $Y_{1}$ is centered and non-centered is not surprising since in the former case $\left|Y_{t}\right|$ grows roughly like $\sqrt{t}$ whereas in the latter case $\left|Y_{t}\right|$ grows like $t$. The persistence exponent of $X \circ|Y|$ is given by the product of the persistence exponent of $X$ and typical growth rate of $Y$. This can be seen in different situations, too, for example if $X$ is an FBM. As in many above statements, the question of replacing the exponential moment condition by the sole assumption of finite variance remains open. is

We conclude this paragraph in mentioning some results for $\operatorname{AR}(p)$ processes, that

$$
X_{n}=a_{1} X_{n-1}+\ldots+a_{p} X_{n-p}+Y_{n},
$$

where $\left\{Y_{n}, n \geq 1\right\}$ is a sequence of i.i.d. random variables and $X_{n}=0$ for $n \leq 0$. The first and already non-trivial question here is to determine for what values of the parameters $a_{1}, \ldots, a_{p}$ the persistence probability (a) converges to some positive constant, (b) decays polynomially, or (c) decays faster than any polynomial (in which case it is usually exponentially small). To do so, one first has to notice, that $X$ can be represented as $X_{n}=\sum_{k=1}^{n} c_{n-k} Y_{k}$ with some $\left(c_{n}\right)$ which depend on the parameters $a_{1}, \ldots, a_{p}$ via the solutions of some characteristic polynomial. The reason for the behavior (a) is that the $\left(c_{n}\right)$ tend to infinity exponentially fast and thus the first few $Y_{n}$ decide the sign of all $X_{n}$. For (c) basically two different main reasons appear: the $\left(c_{n}\right)$ may tend to zero exponentially fast, which means that $X$ is essentially a stationary AR(1) process and one obtains exponential decay of the persistence probability; or the modulus of $\left(c_{n}\right)$ may tend to infinity while the sign is alternating, in which case the sign of $X_{n}$ is determined essentially by the sign of $Y_{n}$ for all $n$ and one basically obtains an independent sequence again leading to exponential decay. The polynomial behaviour is possible only on the critical line between behavior (a) and (c) and corresponds to $\left(c_{n}\right)$ tending to a positive constant. For $p=1$, this is particularly easy: part (a) corresponding to $a_{1}>1$, part (b) to $a_{1}=1$, and part (c) to $a_{1}<1$ (since $c_{n}=a_{1}^{n}$, the above-mentioned distinction happens for $a_{1} \in(-1,1)$ and $\left.a_{1}<-1\right)$. For $p=2$ one obtains the critical curve

$$
\left\{a_{1}=0, a_{2}>1\right\} \cup\left\{a_{1} \in[0,2], a_{1}+a_{2}=1\right\} \cup\left\{a_{1}>2, a_{1}^{2}+4 a_{2}=0\right\}
$$

for the parameter values $\left(a_{1}, a_{2}\right)$ that separates (a) and (c). This question was studied in [71] for $p=1$ and in [11] for general $p$ with a focus on $p=2$. Determining the explicit rate of exponential decay on the region (c) is open in all interesting cases (even for $p=1$ ) and seems to be challenging. Also see [60,28]. 


\section{Some connections with physics}

\subsection{Regular points of inviscid Burgers equation with self-similar initial data}

The statistical study of the one-dimensional Burgers equation

$$
\partial_{t} u+u \partial_{x} u=v \partial_{x x} u
$$

with viscosity $v>0$ and an initial condition $u_{0}(x):=u(0, x)=X_{x}$ given by a selfsimilar stochastic process $\left\{X_{x}, x \in \mathbb{R}\right\}$ has been initiated in the papers [79, 83]. Though this equation is accorded to be an unrealistic physical model for turbulence, the competition between the irregularities of $X$ and the irregularities generated by (4.1) remains an interesting mathematical study. In the inviscid limit $v=0$, the Hopf-Cole solution to (4.1) is given by

$$
u(t, x)=\frac{x-a(t, x)}{t}
$$

for every $t>0, x \in \mathbb{R}$, where $a(t, x)=\max \left\{y \in \mathbb{R}, \dot{C}_{t}(y) \leq x t^{-1}\right\}$ and $\dot{C}$ is the rightderivative of the convex minorant of the function

$$
F_{t}: y \mapsto \int_{0}^{y}\left(X_{x}+x t^{-1}\right) \mathrm{d} x
$$

This variational formula is obtained in considering the explicit solution to (4.1) which can be obtained for $v>0$ and letting $v \rightarrow 0$ - see $[79,14,53,83]$ for details. Notice that $a(t, x)$ is well-defined only if

$$
|x|^{-1} X_{x} \rightarrow 0 \text { a.s. when } x \rightarrow \pm \infty .
$$

The function $x \mapsto a(t, x)$ is right-continuous but not continuous in general, and the so-called Lagrangian regular points at time $t>0$ are defined as the set

$$
\mathscr{L}_{t}=\overline{\{a(t, x), x \in \mathbb{R} \text { and } a(t, x-)=a(t, x)\}}
$$

which consists of the points where $F_{t}$ equals its convex minorant. In physical terms, the set $\mathscr{L}_{t}$ describes the initial locations of the particles which have not been shocked up to time $t$. It is easy to see that when $X$ is a self-similar process, the map $t \mapsto$ $\mathscr{L}_{t}$ has also some self-similarity which makes the a.s. Hausdorff dimension of $\mathscr{L}_{t}$ independent of $t>0$. Setting $\mathscr{L}=\mathscr{L}_{1}$ and "Dim" for "Hausdorff dimension", the following is stated in [79]:

Conjecture 10 (Aurell-Frisch-She) Suppose that $X$ is the fractional Brownian motion with Hurst parameter $H \in(0,1)$. Then $\operatorname{Dim} \mathscr{L}=H$ a.s. 
Notice that in the above, the fractional Brownian motion is defined over the whole $\mathbb{R}$, and coincides with the two-sided Brownian motion when $H=1 / 2$. This conjecture remains open in general, but the following has been shown:

Theorem 4.1 (Handa-Sinai, Bertoin). Suppose that $X$ is the two-sided Brownian motion. Then $\operatorname{Dim} \mathscr{L}=1 / 2$ a.s.

This result was first stated in [83], although no strict proof is given therein for the lower bound $\operatorname{Dim} \mathscr{L} \geq 1 / 2$ a.s. A simple argument based on integration by parts and Frostman's lemma is presented in [48], which yields the general lower bound $\operatorname{Dim} \mathscr{L} \geq H$ a.s. when $X$ is the fractional Brownian motion with Hurst parameter $H$. In [14], the exact computation of the Hausdorff dimension follows as a simple corollary to the more general result that $x \mapsto a(1, x)$ has stationary and independent increments with explicit Laplace transform. This result extends to Lévy processes with no positive jumps satisfying the growth condition (4.2). In particular one has the

Theorem 4.2 (Bertoin). Suppose that $X$ is a two-sided $\alpha$-stable spectrally negative Lévy process with index $\alpha \in(1,2)$. Then $\operatorname{Dim} \mathscr{L}=1 / \alpha$ a.s.

We now briefly describe the link between an upper bound for Dim $\mathscr{L}$ and the computation of certain persistence exponents, in the self-similar framework. This is the original argument of [83] for Brownian motion and it extends to fractional Brownian motion [70] or $\alpha$-stable Lévy processes [81]. Specifically, setting

$$
\hat{\mathscr{L}}=\left\{a \in \mathbb{R}, \int_{0}^{y}\left(X_{x}+x\right) \mathrm{d} x \geq \int_{0}^{a}\left(X_{x}+x\right) \mathrm{d} x+(y-a)\left(X_{a}+a\right), \forall y \in \mathbb{R}\right\},
$$

Sinai's remarkable and simple observation is that up to some countable set, one has

$$
\mathscr{L} \subseteq \hat{\mathscr{L}} \quad \text { a.s. }
$$

On the other hand, the Borel-Cantelli lemma and some elementary inequalities - see Lemma 1 in [70] and [81] pp. 742-745 for all details - show that if

$$
\mathbb{P}\left[\int_{0}^{y}\left(X_{x}+x\right) \mathrm{d} x \geq-\delta^{1+H}, \forall y \in[-1,1]\right] \leq \delta^{1-K+o(1)}
$$

as $\delta \rightarrow 0$, where $H$ is $X$ 's self-similarity index and $K \in[0,1]$, then $\operatorname{Dim} \overline{\mathscr{L}} \leq K$ a.s. Hence, the "two-sided" persistence probability evaluation (4.3) entails the upper bound $\operatorname{Dim} \mathscr{L} \leq H$ a.s. The upper asymptotic inequality (4.3) is shown in [82] for Brownian motion and the above method is used in [83] to obtain the upper bound in Theorem 4.1.

If $X=B^{H}$ is the fractional Brownian motion, then the drift appearing in (4.3) can be removed by quasi-invariance and some analysis, and one sees by symmetry and self-similarity that the required estimate to get the upper bound in Conjecture 10 is

$$
\mathbb{P}\left[\int_{0}^{y} B_{x}^{H} \mathrm{~d} x \leq 1, \forall y \in[-t, t]\right] \leq t^{1-H+o(1)}, \quad t \rightarrow+\infty .
$$


The above estimate is formulated as a conjecture in [70,68], with an equality instead of the inequality. Notice that this latter problem is independent of Conjecture 8 since the increments of $B^{H}$ are correlated. Actually, even the sole existence of the persistence exponent for integrated double-sided fractional Brownian motion has not yet been established.

If $X$ is a two-sided $\alpha$-stable Lévy process with $\alpha \in(1,2)$, there is no quasiinvariance argument. But the fact that $\alpha>1$ and the bare-hand analysis performed in [81] make believe that the drift appearing in (4.3) can also be removed. By selfsimilarity and independence of the positive and negative increments of $Z$ the inequality (4.3) would then amount to

$$
\mathbb{P}\left[\hat{T}_{1}>t\right] \leq t^{(1-K) / 2+o(1)}
$$

at infinity, where $\hat{T}_{1}$ is the first-passage time at 1 of the integral of $\hat{Z}=-Z$. In particular the validity of Conjecture 3 would lead to $\operatorname{Dim} \mathscr{L} \leq \rho$ a.s. where $\rho=$ $\mathbb{P}\left[Z_{1}>0\right]$ is the positivity parameter of $Z$ (recall that $\rho \in[1-1 / \alpha, 1 / \alpha]$ for $\alpha \in$ $(1,2))$. In view of the above theorem, it is natural to state the

Conjecture 11 Suppose that $X$ is a two-sided $\alpha$-stable Lévy process with $\alpha \in(1,2)$ and positivity parameter $\rho=\mathbb{P}\left[Z_{1}>0\right]$. Then $\operatorname{Dim} \mathscr{L}=\rho$ a.s.

This Hausdorff dimension depending on the positivity parameter and not on the self-similarity index is different from the value $1 / \alpha$ which had been conjectured in [53] through multifractal analysis. The invalidity of the latter when $\alpha$ is close to 1 was proved in [81] with the help of the above Sinai's approach:

Theorem 4.3 (Simon). For every $c<1$ there exists $\alpha_{0}>1$ such that for every $\alpha \in$ $\left(1, \alpha_{0}\right)$ and every $\rho \in[1-1 / \alpha, c \wedge 1 / \alpha]$, if $X$ is a two-sided $\alpha$-stable Lévy process with positivity parameter $\rho$, then $\operatorname{Dim} \mathscr{L}<1 / \alpha$ a.s.

We conclude this paragraph in mentioning that the lower bound in Conjecture 11, which should be obtained by different and yet unknown arguments, would lead by comparison to the lower bound of Conjecture 3 in the case $\alpha>1$.

\subsection{Positivity of random polynomials and diffusion equation}

A classical question dating back to the beginning of probability theory is to understand the distribution of the roots of random polynomials. Consider

$$
P_{n}(X)=\sum_{i=0}^{n-1} \xi_{i} X^{i}
$$

with large even degree where $\left\{\xi_{i}, i \geq 0\right\}$ is some i.i.d. sequence and $X$ the deterministic variable, and set $N_{n}$ for the number of its real roots. Among other results, the following was recently obtained in [31]: 
Theorem 4.4 (Dembo-Poonen-Shao-Zeitouni). Suppose that $\xi_{1}$ is centered and has polynomial moments of all order. Then

$$
\mathbb{P}\left[N_{2 n+1}=0\right]=n^{-4 b+o(1)}
$$

where $b=-\lim _{t \rightarrow \infty} t^{-1} \log \mathbb{P}\left[Y_{s}<0, \forall s \in[0, t]\right]$, with $Y$ the centered $\mathrm{GSP}$ with correlation $\mathbb{E}\left[Y_{0} Y_{t}\right]=1 / \cosh (t / 2)$.

In the above, the exact value of $b$ is unknown and numerical simulations suggest $4 b=0.79 \pm 0.03$ - see [31]. It is remarkable that this constant $b$ relates to $n$ times integrated Brownian motion. We saw indeed in Paragraph 3.3 that $b=\lim _{n \rightarrow+\infty} \theta(n)$ where $\theta(n)$ is the persistence exponent of the process

$$
t \mapsto \frac{1}{n !} \int_{0}^{t}(t-s)^{n} \mathrm{~d} B_{s}, \quad t \geq 0 .
$$

The problem of computing $\theta(n)$ for $n>1$ is believed to be very challenging. Numerical simulations [64] suggest $\theta(2)=0.231 \pm 0.01$.

Let us give some insight on the proof of the above result. The hard part is to show (4.4) when $\xi_{1} \sim \mathscr{N}(0,1)$. The general case follows by strong approximation, whence the assumption made on the moments, but notice that it is also conjectured in [31] that (4.4) should hold under the sole condition that $\xi_{1}$ is centered and has finite variance. When $\xi_{1} \sim \mathscr{N}(0,1)$, the process $x \rightarrow P_{n}(x)$, which is the so-called Kac's polynomial, is centered Gaussian with covariance $\mathbb{E}\left[P_{n}(x) P_{n}(y)\right]=1+\cdots+(x y)^{n-1}$. Its correlation function is given by

$$
\left|\frac{(x y)^{n}-1}{x y-1}\right| \sqrt{\left|\frac{\left(x^{2}-1\right)\left(y^{2}-1\right)}{\left(x^{2 n}-1\right)\left(y^{2 n}-1\right)}\right|}
$$

for every $x, y \neq \pm 1$. This function is invariant under the transformations $(x, y) \mapsto$ $(-x,-y)$ and $(x, y) \mapsto(1 / x, 1 / y)$, and an involved argument based on Slepian's lemma shows that

$$
\mathbb{P}\left[N_{2 n+1}=0\right]=\left(\mathbb{P}\left[N_{2 n+1}^{[0,1]}=0\right]\right)^{4+o(1)}
$$

where $N_{n}^{[0,1]}$ is the number of roots of $P_{n}$ on $[0,1]$. The link between $N_{2 n+1}^{[0,1]}$ for large $n$ and the zero-crossings of $Y$ is established after changing the variable $x=e^{-t}$ and isolating the contributions for small $t$. The latter is the crucial step, since it follows from the singularities of the correlation function that the density of the real roots of $P_{n}$ around \pm 1 is very big for large $n$. Notice that the link between $Y$ and $N_{n}$ is rather easily understood at the expectation level: a classical formula due to Kac - see [31] for details - yields

$$
\mathbb{E}\left[N_{2 n+1}^{[0,1]}\right] \sim \frac{1}{2 \pi} \log n
$$


whereas the Itô-Rice's formula shows that if $N_{t}$ is the number of zero-crossings of $Y$ on $[0, t]$, then $\mathbb{E}\left[N_{t}\right] \sim t /(2 \pi)$. The problem of evaluating $\mathbb{P}\left[N_{2 n+1}^{[0,1]}=0\right]$ is however much more intricate than the sole estimation of $\mathbb{E}\left[N_{2 n+1}^{[0,1]}\right]$, in analogy with what happens for the zero-crossings of Gaussian stationary processes.

It is also a remarkable fact that the above constant $b$ appears as the persistence exponent of another, seemingly disconnected random evolution phenomenon, which is studied in $[63,78,77]$. Consider the heat equation on $\mathbb{R}^{d}$

$$
\frac{\partial u_{d}}{\partial t}=\Delta u_{d}
$$

with random initial condition $u_{d}(x, 0)=\dot{W}(x)$ a $d$-dimensional white noise. Integrating along the heat kernel, it is easy to see by linearity that for every $x \in \mathbb{R}^{d}$ the solution $t \mapsto u_{d}(x, t)$ to $(4.5)$ is a $(-d / 4)$-self-similar centered Gaussian process with covariance function

$$
\mathbb{E}\left[u_{d}(t, x) u_{d}(s, x)\right]=\frac{1}{(\pi(t+s))^{d / 2}} .
$$

In particular the law of $\left\{u_{d}(t, x), t>0\right\}$ does not depend on $x$, which is also clear from the white noise initial condition. The Lamperti transformation $t \mapsto$ $(2 \pi)^{1 / 4} e^{t / 4} u_{d}\left(x, e^{t}\right)$ is a centered GSP with correlation function $1 /(\cosh (t / 2))^{d / 2}$, and this GSP coincides with $Y$ for $d=2$. For every $d \geq 1$, the standard subadditivity argument and Slepian's lemma yield the existence of $\lambda_{d}>0$ such that

$$
\mathbb{P}\left[u_{d}(s, x)<0, \forall s \in[1, t]\right]=t^{-\lambda_{d}+o(1)}
$$

and one has $b=\lambda_{2}$. In [63], an empirical approach using independent interval approximation is described, proposing $\lambda_{d}$ as the first zero on the negative axis of the function

$$
x \mapsto 1+\sqrt{\frac{2}{d}}\left(\pi x-2 x^{2} \int_{0}^{\infty} e^{-x t} \sin ^{-1}\left[1 /(\cosh (t / 2))^{d / 2}\right] \mathrm{d} t\right),
$$

which yields the numerical values $\lambda_{1}=0.1207, \lambda_{2}=0.1862$ and $\lambda_{3}=0.2358$. In the above formula, the function $d \mapsto \lambda_{d}$ is increasing, which is somehow in heuristic accordance with the fact that in the first-order expansion

$$
1 /(\cosh (t / 2))^{d / 2}=1-\frac{d t^{2}}{16}+o\left(t^{2}\right),
$$

the coefficient $d \mapsto d / 16$ also increases. In [78] it is argued that $\lambda_{d} \sim c \sqrt{d}$ at infinity, for some constant $c>0$. The paper [78] also establishes for every $d \geq 1$ a general connection between the survival analysis of the equation (4.5) and the positivity of a family of random polynomials defined as 


$$
P_{n}^{d}(X)=\xi_{0}+\sum_{i=1}^{n-1} i^{(d-2) / 4} \xi_{i} X^{i}
$$

where $\left\{\xi_{i}, i \geq 0\right\}$ is a i.i.d. sequence of $\mathscr{N}(0,1)$ random variables and $X$ is the deterministic variable. Setting $N_{n}^{d}$ for the number of its real roots, it is argued in [78] that

$$
\mathbb{P}\left[N_{2 n+1}^{d}=0\right]=n^{-2\left(\lambda_{2}+\lambda_{d}\right)+o(1)} .
$$

The results are justified in the recent paper [30], where the connection between the persistence of the Gaussian process $u_{d}$ defined in (4.6) and the random polynomials (4.7) for Gaussian $\xi_{i}$ is proved rigorously. Further, the notion of a solution to the heat equation (4.5) is made rigorous. It would be interesting to obtain again a universality result similar to Theorem 4.4 in the sense that (4.8) holds independent of the law of the $\xi_{i}$. Further, [30] establishes connections of other types of random polynomials and the corresponding Gaussian processes (also considered in [78]). Also here it would be interesting to obtain a universality result.

\subsection{Wetting models with Laplacian interactions}

Let $f$ be a bounded and everywhere positive probability density over $\mathbb{R}$, centered and having finite variance. Introduce the Hamiltonian $\mathscr{H}_{[a, b]}(\varphi)$, defined for $a, b \in \mathbb{Z}$ with $b-a \geq 2$ and for $\varphi:\{a, \ldots, b\} \rightarrow \mathbb{R}$ by

$$
\mathscr{H}_{[a, b]}(\varphi)=\sum_{n=a+1}^{b-1} V\left(\Delta \varphi_{n}\right)
$$

where $V=-\log (f)$ is the potential and

$$
\Delta \varphi_{n}=\left(\varphi_{n+1}-\varphi_{n}\right)-\left(\varphi_{n}-\varphi_{n-1}\right)=\varphi_{n+1}-2 \varphi_{n}+\varphi_{n-1}
$$

is the discrete Laplacian on $\mathbb{Z}$. The free pinning model with Laplacian interaction is the probability measure on $\mathbb{R}^{N-1}$ defined by

$$
\mathbb{P}_{0, N}^{p}\left(\mathrm{~d} \varphi_{1}, \ldots, \mathrm{d} \varphi_{N-1}\right)=\frac{\exp \left(-\mathscr{H}_{[-1, N+1]}(\varphi)\right)}{\mathscr{Z}_{0, N}^{p}} \mathrm{~d} \varphi_{1} \ldots \mathrm{d} \varphi_{N-1}
$$

where $\mathscr{Z}_{0, N}^{p}$ is the normalization constant which is called the partition function, and where the boundary conditions are given by $\varphi_{-1}=\varphi_{0}=\varphi_{N}=\varphi_{N+1}=0$. This probability measure models a certain $(1+1)$-dimensional field (viz. a linear chain $\left.\left\{\left(n, \varphi_{n}\right), n=0 \ldots N\right\}\right)$ with zero boundary conditions and whose interacting structure is described by the discrete Laplacian and the potential $V$. This chain can be viewed as an example of a discrete random polymer in $(1+1)$-dimension. 
The free pinning model with gradient interaction, where $\Delta$ is replaced by the discrete gradient $\nabla \varphi_{n}=\varphi_{n+1}-\varphi_{n}$ and where the boundary conditions are $\varphi_{0}=\varphi_{N}=0$, has been well studied in the literature and has a natural interpretation in terms of random bridges with increment density given by $f$. The model with Laplacian interaction has exactly the same interpretation in terms of integrated random bridges. Specifically, one can easily show - see Section 2 in [24] for details - that $\mathbb{P}_{0, N}^{p}$ is, with the notations of Section 3.1, the law of an integrated random walk $\left\{A_{n}=S_{1}+\cdots+S_{n}, n=1 \ldots N-1\right\}$ conditioned on $A_{N}=A_{N+1}=0$, with increment density given by $f$. The partition function $\mathscr{Z}_{0, N}^{p}$ is then the value at $(0,0)$ of the density of $\left(S_{N+1}, A_{N+1}\right)$. Notice that both above free pinning models have natural counterparts in continuous time in the context of semiflexible polymers. The gradient interacting case corresponds to directed polymers, whereas the Laplacian interacting case corresponds to polymers with non-zero bending energy - see [23] for details.

The connection with persistence of integrated random bridges is made in considering the corresponding wetting model with Laplacian interactions, which is the probability measure on $\mathbb{R}^{N-1}$ defined by

$$
\begin{aligned}
\mathbb{P}_{0, N}^{w}\left(\mathrm{~d} \varphi_{1}, \ldots, \mathrm{d} \varphi_{N-1}\right) & =\mathbb{P}_{0, N}^{p}\left(\mathrm{~d} \varphi_{1}, \ldots, \mathrm{d} \varphi_{N-1} \mid \varphi_{1} \geq 0, \ldots, \varphi_{N-1} \geq 0\right) \\
& =\frac{\exp \left(-\mathscr{H}_{[-1, N+1]}(\varphi)\right)}{\mathscr{Z}_{0, N}^{w}} \mathbf{1}_{\left\{\varphi_{1} \geq 0, \ldots, \varphi_{N-1} \geq 0\right\}} \mathrm{d} \varphi_{1} \ldots \mathrm{d} \varphi_{N-1}
\end{aligned}
$$

where $\mathscr{Z}_{0, N}^{w}$ is the normalization constant, and with the same boundary conditions $\varphi_{-1}=\varphi_{0}=\varphi_{N}=\varphi_{N+1}=0$. In this model, the discrete random polymer is in the presence of a one-dimensional hard wall at zero which forces it to stay non negative. From the very definition, one sees that $\mathbb{P}_{0, N}^{W}$ is the law of the above integrated random walk $\left\{A_{n}, n=1 \ldots N-1\right\}$ whose increment has the density $f$, and conditioned on $\Omega_{N-1}^{+} \cap\left\{A_{N}=A_{N+1}=0\right\}$ with the notation $\Omega_{N-1}^{+}=\left\{A_{1} \geq 0, \ldots, A_{N-1} \geq 0\right\}$. It is then easily shown that the partition function is given by

$$
\mathscr{Z}_{0, N}^{w}=\mathbb{P}\left[\Omega_{N-1}^{+} \mid A_{N}=A_{N+1}=0\right] f_{N}(0,0)
$$

where $f_{N}(0,0)$ is the value at $(0,0)$ of the density of $\left(S_{N+1}, A_{N+1}\right)$. As a consequence of a local limit theorem - see Section 2 in [24] for details - it can be shown that $f_{N}(0,0) \sim c N^{-2}$ at infinity for some explicit constant $c>0$. Hence the behavior of $\mathscr{Z}_{0, N}^{w}$ for $N$ large, which has some importance in physics, is specified by the persistence probability

$$
\mathbb{P}\left[\Omega_{N-1}^{+} \mid A_{N}=A_{N+1}=0\right] .
$$

The latter quantity has also some independent interest as a question about entropic repulsion - see all the references listed in [24] for more on this subject, and the following is stated in [24]:

Conjecture 12 (Caravenna-Deuschel) With the above notations, one has

$$
\mathbb{P}\left[\Omega_{N-1}^{+} \mid A_{N}=A_{N+1}=0\right] \asymp N^{-1 / 2}
$$


for every centered increment law $\mu$ having finite variance.

This conjecture is related to integrated random walks considered in Section 3.1 since the event $\left\{\Omega_{N-1}^{+} \mid A_{N}=A_{N+1}=0\right\}$ can be decomposed into $\left\{A_{1} \geq 0, \ldots, A_{N / 2} \geq\right.$ $\left.0 \mid A_{N}=A_{N+1}=0\right\} \cap\left\{A_{N / 2+1} \geq 0, \ldots, A_{N-1} \geq 0 \mid A_{N}=A_{N+1}=0\right\}$, the intersection of two roughly independent events with roughly the same probability $\mathbb{P}\left[A_{1} \geq\right.$ $\left.0, \ldots, A_{N / 2} \geq 0\right]$, a quantity which should behave like $N^{-1 / 4}$. Notice that in the context of semiflexible polymers, a continuous couterpart of $\Omega_{N-1}^{+}$in the case when $\mu$ is Gaussian was investigated (without conditioning) in [23], where the estimate (3.3) is proved. In [24], the following weak bounds are obtained

$$
\frac{c}{N^{c_{-}}} \leq \mathbb{P}\left[\Omega_{N-1}^{+} \mid A_{N}=A_{N+1}=0\right] \leq \frac{C}{(\log N)^{c_{+}}}
$$

for some constants $c, C, c_{-}>0$ and $c_{+}>1$. The lower bound entails that the free energy vanishes:

$$
\lim _{N \rightarrow+\infty} \frac{1}{N} \log \mathscr{Z}_{0, N}^{w}=0,
$$

whereas the fact that $c_{+}>1$ in the upper bound is crucial to show that the phase transition of the wetting model with reward, which is simply the value of the positive parameter $\varepsilon$ after which the free energy of the probability measure

$$
\frac{\exp \left(-\mathscr{H}_{[-1, N+1]}(\varphi)\right)}{\mathscr{Z}_{\varepsilon, N}^{w}} \prod_{n=1}^{N-1}\left(\varepsilon \delta_{0}\left(\mathrm{~d} \varphi_{n}\right)+\mathbf{1}_{\left\{\varphi_{n} \geq 0\right\}} \mathrm{d} \varphi_{n}\right)
$$

becomes positive, is of first order. In [24], to which we again refer for more details, it is mentioned that Conjecture 12 would yield some further path results for the wetting model with reward at criticality.

Recently, there have been two advances in this direction. First, [6] show Conjecture 12 in the case of the simple random walk and integrated simple random walk:

Theorem 4.5 (Aurzada-Dereich-Lifshits). Let $\left\{X_{i}, i \geq 0\right\}$ be an i.i.d. sequence of Bernoulli random variables, and consider the simple random walk $S_{n}:=\sum_{i=1}^{n} X_{i}$ and the integrated random walk $A_{n}:=\sum_{i=1}^{n} S_{i}$. Then

$$
\mathbb{P}\left[A_{1} \geq 0, \ldots, A_{4 n} \geq 0 \mid S_{4 n}=0, A_{4 n}=0\right] \asymp n^{-1 / 2} .
$$

Further, it is mentioned in [34] that the techniques can be used to show the full Conjecture 12 under the condition of finite $(2+\delta)$ moment for some $\delta>0$. Presumably, the techniques will be different to [6] and it would be very interesting to study this question and its implications for the wetting models at criticality. Similarly to the above theorem, the case of Gaussian $\left(X_{i}\right)$ was studied very recently by [45] and the corresponding result is shown (up to a log factor in the lower bound). 


\subsection{Other physical applications}

\subsubsection{Spatial persistence for fluctuating interfaces}

A fluctuating interface is a function $h: \mathbb{R}^{+} \times \mathbb{R}^{d} \rightarrow \mathbb{R}$ evolving in time, with dynamics governed by a certain random equation. The problem of spatial persistence concerns the probability $p(l)$ that such a fluctuating interface stays above its initial value over a large distance $l$ from a given point in space. One expects a behavior like $p(l)=l^{-\theta+o(1)}$ for a positive number $\theta$ independent of the direction, which is called the spatial persistence of the interface. In [64], this question is adressed for the Gaussian interface $h(t, x)$ solution to the equation

$$
\frac{\partial h}{\partial t}=-(-\Delta)^{z / 2} h+\xi
$$

where $\Delta$ is the $d$-dimensional Laplacian, $\xi$ a space-time white noise with zero mean, and $z>d$ some fractional parameter, and it is shown with heuristic arguments based on Fourier inversion that if $\rho=(z-d+1) / 2$, then the fractional derivative in any direction $x_{1}$

$$
\frac{\partial^{\rho} h}{\partial x_{1}^{\rho}}=\frac{\partial}{\partial x_{1}^{[\rho]+1}}\left(\frac{1}{\Gamma(2-\alpha)} \int_{0}^{x_{1}} h\left(t, y, x_{2}, \ldots, x_{d}\right)\left(x_{1}-y\right)^{1-\alpha} \mathrm{d} y\right)
$$

where we have decomposed $\rho=[\rho]+\alpha$ into integer and fractional parts, is a onedimensional white noise. This relates the spatial persistence probability $p(l)$ to the persistence probability of the fractionally integrated Lévy process

$$
A_{t}^{\rho}=\frac{1}{\Gamma(\rho)} \int_{0}^{t}(t-s)^{\rho-1} \mathrm{~d} B_{s} .
$$

In [64] two different regimes are considered. The coarsening one, where the reference point is fixed, yields a spatial persistence exponent $\theta=\theta(\rho)$ with the notations of Paragraph 3.3. The stationary one, where the reference point is sampled uniformly from the ensemble of steady state configurations, yields from (3.6) a spatial persistence exponent $\theta=(1-\rho)_{+}$. In the coarsening regime, this entails that the zero crossings of Gaussian interfaces governed by (4.9) undergo a morphological transition at $z=d+2$, because then $\rho=3 / 2$.

\subsubsection{Clustering of sticky particles at critical time}

In this last paragraph we consider a random walk $\left\{S_{n}, n \geq 1\right\}$ with positive increments having expectation $\mathbb{E}\left[S_{1}\right]=1$. If $n$ particles are fixed at the respective positions $i^{-1} S_{i}, i=1 \ldots n$ with zero initial speed and then move according to the laws of gravitational attraction, these particles end up in sticking together with conservation of mass and momentum, forming new particles called clusters. One is then 
interested in the number of clusters $K_{n}(t) \in[1, \ldots, n]$ viz. the total number of particles present at time $t \geq 0$. This is a so-called sticky particle model, which is for $n$ large connected to the inviscid Burgers equation with random initial data (coupled with some scalar transport equation, see [22] for details). This is also an aggregation model having connections with astrophysics, and we refer to the introduction of [84] for a clarification of these relations and the complete dissipation of all possible misunderstanding.

The normalization $\mathbb{E}\left[S_{1}\right]=1$ entails that $T \rightarrow 1$ in probability, where $T$ stands for the random terminal time where all particles have aggregated in a single cluster. A more precise result is obtained in [84] in the case when $S_{1}$ has uniform or standard Poissonian distribution, namely that the random function

$$
\frac{K_{n}(t)-n\left(1-t^{2}\right)}{\sqrt{n}}
$$

converges in law to some Gaussian process on the Skorokhod space $\mathscr{D}[0,1-\varepsilon]$ for any $\varepsilon>0$. In particular, the above quantity converges to some Gaussian law at each fixed time $t<1$. The situation is however different at the critical time $t=1$, at least when $S_{1}$ has a standard Poissonian distribution. In this case it can be proved that $K_{n}(1) / \sqrt{n}$ does not converge to zero (the only non-negative Gaussian distribution) as could be expected, and this fact is actually a consequence - see [84] for details of the estimate

$$
\mathbb{P}\left[\min _{i=1, \ldots, n} \sum_{j=1}^{i}\left(\Gamma_{j}-j\right) \geq 0\right] \asymp n^{-1 / 4}
$$

where $\left\{\Gamma_{n}, n \geq 1\right\}$ is a random walk with exponential increments - this latter estimate follows from the main result of [29].

\section{Remarks in press}

After this paper was accepted, a number of works falling into the scope of this survey have appeared. We mention them here only briefly, referring to the papers themselves for more detail.

In Section 2, the classical framework for first passage times over a constant boundary of random walks and Lévy processes is displayed. A novel approach to this framework has been given in [57, 58], together with new and rather general results. The recent papers $[9,10,8,33]$ deal with the less classical framework of first passage time problems over a moving boundary, and provide some tight estimates on the distribution functions.

The Conjectures 3 and 4 presented in Section 3.2 have been disproved in [73], where it is shown that for integrated $\alpha$-stable Lévy processes, the persistence exponent is given by $\theta=\rho /(1+\alpha(1-\rho))$, with the above notation. See also [74] for related results on $n$-th passage times, with the exact logarithmic correction term. 
Because of this result, Conjectures 1 and 2 seem now unplausible, yet not strictly disproved.

The relation between inverse exponential functionals and the persistence probability studied in Section 3.3 is based on Molchan's result [67] for continuous-time processes. In [7], this relation between inverse exponential functionals and the persistence probability is studied for discrete-time processes for the first time. The technique is applied to random walks in random sceneries (in any dimension) and to sums of stationary sequences with long-range dependence. We also mention that [35] studies persistence of additive functionals of Sinai's random walk in random environment obtaining the persistence exponent $\theta=(3-\sqrt{5}) / 2$; where the value comes from a large deviation rate for the number of sign changes.

Finally, a general question connected to Section 3.3 has been studied in [42]: When does a stationary Gaussian process have an exponentially decreasing persistence probability? In the mentioned paper, rather general sufficient conditions are given.

Acknowledgements This work was supported by the DFG Emmy Noether research program and the grant ANR-09-BLAN-0084-01 Autosimilaires. The authors would like to thank R. A. Doney, S. N. Majumdar, A. A. Novikov and the referees for their useful comments.

\section{References}

1. S. Asmussen. Ruin probabilities. Springer-Verlag, New-York, 2000.

2. F. Aurzada. On the one-sided exit problem for fractional Brownian motion. Elec. Comm. Probab. 16, 392-404, 2011.

3. F. Aurzada and C. Baumgarten. Survival probabilities for weighted random walks. ALEA Lat. Am. J. Probab. Math. Stat. 8, 235-258, 2011.

4. F. Aurzada and C. Baumgarten. Persistence of fractional Brownian motion with moving boundaries and applications. J. Phys. A, 46(12):125007, 12, 2013.

5. F. Aurzada and S. Dereich. Universality of the asymptotics of the one-sided exit problem for integrated processes. Ann. Inst. H. Poincaré Probab. Statist. 49, 236-251, 2013.

6. F. Aurzada, S. Dereich, and M. Lifshits. Persistence probabilities for a bridge of an integrated simple random walk. Probab. Math. Statist., 34(1):1-22, 2014.

7. F. Aurzada and N. Guillotin-Plantard. Persistence exponent for discrete-time, time-reversible processes. Preprint, arXiv:1502.06799.

8. F. Aurzada and T. Kramm. The first passage time problem over a moving boundary for asymptotically stable Lévy processes. Preprint, arXiv:1305.1203, Journal of Theoretical Probability, to appear.

9. F. Aurzada and T. Kramm. First exit of Brownian motion from a one-sided moving boundary. In High Dimensional Probability VI: The Banff Volume, number 66 in Progress in Probability, pages 215-219. Springer, 2013.

10. F. Aurzada, T. Kramm, and M. Savov. First passage times of Lévy processes over a one-sided moving boundary. Preprint, arXiv:1201.1118, Markov processes and related fields, to appear.

11. C. Baumgarten. Survival probabilities of autoregressive processes. ESAIM Probab. Stat., 18:145-170, 2014.

12. C. Baumgarten. Survival probabilities of some iterated processes. Probab. Math. Stat., 34(2):293-316, 2014. 
13. J. Bertoin. Lévy Processes. Cambridge University Press, Cambridge, 1996.

14. J. Bertoin. The inviscid Burgers equation with Brownian initial velocity. Comm. Math. Phys. 193 (2), 397-406, 1998.

15. J. Bertoin and R. A. Doney. Spitzer's condition for random walks and Lvy processes. Ann. Inst. H. Poincaré Probab. Statist. 33, 167-178, 1997.

16. N. H. Bingham. Maxima of sums of random variables and suprema of stable processes. $Z$. Wahr. verw. Geb. 26, 273-296, 1973.

17. N. H. Bingham. Fluctuation theory in continuous time. Adv. Appl. Probab. 7, 705-766, 1975.

18. N. H. Bingham. Random walk and fluctuation theory. In: Shanbhag et al. (ed.), Stochastic processes: Theory and methods. Handb. Stat. 19, 171-213, 2001.

19. N. H. Bingham, C. M. Goldie and J. L. Teugels. Regular variation. Cambridge University Press, Cambridge, 1987.

20. A. J. Bray and P. Gonos. Survival of a diffusing particle in a transverse shear flow: a first passage problem with continuously varying persistence exponent. J. Phys. A: Math. Gen. 37, 361-366, 2004

21. A. Bray, S. Majumdar, and G. Schehr. Persistence and first-passage properties in nonequilibrium systems. Advances in Physics, 62(3):225-361, 2013.

22. Y. Brenier and E. Grenier. Sticky particles and scalar conservation laws. SIAM J. Numer. Anal. 35 (6), 2317-2328, 1998.

23. T. W. Burkhardt. Semiflexible polymer in the half-plane and statistics of the integral of the Brownian curve. J. Phys. A: Math. Gen. 26, 1157-1162, 1993.

24. F. Caravenna and J.-D. Deuschel. Pinning and wetting transition for (1+1)-dimensional fields with Laplacian interaction. Ann. Probab. 36 (6), 2388-2433, 2008.

25. F. Castell, N. Guillotin-Plantard, F. Pène, and B. Schapira. On the one-sided exit problem for stable processes in random scenery. Electron. Commun. Probab., 18(33):1-7, 2013.

26. F. Castell, N. Guillotin-Plantard, and F. Watbled. Persistence exponent for random processes in Brownian scenery. Preprint, arXiv:1407.0364.

27. M. Constantin, C. Dasgupta, P. Punyindu Chatraphorn, S. N. Majumdar and S. Das Sarma. Persistence in nonequilibrium surface growth. Phys. Rev. E 69, 061608 (22 pages), 2004.

28. A. Dembo and J. Ding. Persistence probabilities for auto-regressive sequences. Preprint, http://statweb.stanford.edu/ adembo/.

29. A. Dembo, J. Ding, and F. Gao. Persistence of iterated partial sums. Ann. Inst. Henri Poincaré Probab. Stat., 49(3):873-884, 2013.

30. A. Dembo and S. Mukherjee. No zero-crossings for random polynomials and the heat equation. Ann. Probab., 43:85-118, 2015.

31. A. Dembo, B. Poonen, Q.-M. Shao and O. Zeitouni. Random polynomials having few or no real zeros. J. Am. Math. Soc. 15 (4), 857-892, 2002.

32. D. Denisov and V. Wachtel. Random walks in cones. Preprint, arXiv:1110.1254, Ann. Probab., to appear.

33. D. Denisov and V. Wachtel. Exact asymptotics for the instant of crossing a curve boundary by an asymptotically stable random walk. Preprint, arXiv:1403.5918.

34. D. Denisov and V. Wachtel. Exit times for integrated random walks. Preprint, arXiv:1207.2270, Ann. Inst. H. Poincare Probab. Statist., to appear.

35. A. Devulder. Persistence of some additive functionals of Sinai's walk. Preprint, arXiv:1402.2267.

36. A. Devulder, Z. Shi and T. Simon. The lower tail problem for integrated symmetric stable processes. Unpublished manuscript, 2005.

37. R. A. Doney. Spitzer's condition and ladder variables in random walks. Probab. Theory Relat. Fields 101 577-580, 1995.

38. R. A. Doney. On the asymptotic behaviour of first passage times for transient random walks. Probab. Theory Relat. Fields 81, 239-246, 1989.

39. R. A. Doney. Fluctuation theory for Lévy processes. Lect. Notes Math. 1897. Springer-Verlag, Berlin, 2007.

40. H. Dym and H.-P. McKean. Fourier series and integrals. Academic Press, New-York, 1972. 
41. S. Engelke and J. H. C. Woerner. A unifying approach to fractional Lévy processes. Stoch. Dyn. 13 (2), 1250017 (19 pages), 2013.

42. N. Feldheim and O. N. Feldheim. Long gaps between sign-changes of gaussian stationary processes. Preprint, arXiv:1307.0119, International Mathematics Research Notices, to appear.

43. W. Feller. An introduction to probability theory and its applications. Vol II. Wiley, New-York, 1971.

44. D. A. Freedman. Survival analysis: a primer. The American Statistician. 62 (2), 110-119, 2008.

45. F. Gao, Z. Liu, and X. Yang. Conditional persistence of Gaussian random walks. Electron. Commun. Probab., 19(70), 2014.

46. M. Goldman. On the first passage of the integrated Wiener process. Ann. Math. Statist. 42 (6), 2150-2155, 1971.

47. P. Groeneboom, G. Jongbloed, and J. A. Wellner. Integrated Brownian motion, conditioned to be positive. Ann. Probab. 27, 1283-1303, 1999.

48. K. Handa. A remark on shocks in inviscid Burgers' turbulence. In Fitzmaurice et al. (ed): Nonlinear waves and weak turbulence with applications in oceanography and condensed matter physics. Prog. Nonlinear Differ. Equ. Appl. 11, 339-345, 1993.

49. Y. Isozaki. Asymptotic estimates for the distribution of additive functionals of Brownian motion by the Wiener-Hopf factorization method. J. Math. Kyoto Univ. 36 (1), 211-227, 1996.

50. Y. Isozaki and S. Kotani. Asymptotic estimates for the first hitting time of fluctuating additive functionals of Brownian motion. Sémin. Probab. XXXIV, 374-387, 2000.

51. Y. Isozaki and S. Watanabe. An asymptotic formula for the Kolmogorov diffusion and a refinement of Sinai's estimates for the integral of Brownian motion. Proc. Japan Acad., Ser. A 70 (9), 271-276, 1994.

52. N. C. Jain and W. E. Pruitt. Lower tail probability estimates for subordinators and nondecreasing random walks. Ann. Probab. 15 (1), 75-101, 1987.

53. A. W. Janicki and W. A. Woyczynski. Hausdorff dimension of regular points in stochastic Burgers flows with Lévy $\alpha$-stable initial data. J. Stat. Phys. 86 (1-2), 277-299, 1997.

54. J. H. B. Kemperman. The Passage Problem for a Stationary Markov Chain. University of Chicago Press, Chicago, 1961.

55. H. Kesten. Sums of independent random variables without moment conditions. Ann. Math. Stat. 43 (3), 701-732, 1972.

56. A. N. Kolmogoroff. Zufällige Bewegungen (zur Theorie der brownschen Bewegung). Ann. of Math. (2) 35 (1), 116-117, 1934.

57. M. Kwaśnicki, J. Małecki, and M. Ryznar. Suprema of Lévy processes. Ann. Probab., 41(3B):2047-2065, 2013.

58. M. Kwaśnicki, J. Małecki, and M. Ryznar. First passage times for subordinate Brownian motions. Stochastic Process. Appl., 123(5):1820-1850, 2013.

59. A. Lachal. Sur le premier instant de passage de l'intégrale du mouvement brownien. Ann. Inst. Henri Poincaré, Probab. Stat. 27 (3), 385-405, 1991.

60. H. Larralde. A first passage time distribution for a discrete version of the Ornstein-Uhlenbeck process. J. Phys. A, 37(12):3759-3767, 2004.

61. W. V. Li and Q.-M. Shao. A normal comparison inequality and its application. Probab. Theory Relat. Fields 122 (1), 494-508, 2002.

62. W. V. Li and Q.-M. Shao. Lower tail probabilities for Gaussian processes. Ann. Probab. 32 (1), 216-242, 2004.

63. S. N. Majumdar. Persistence in nonequilibrium systems. Current Science 77 (3), 370-375, 1999.

64. S. N. Majumdar and A. J. Bray. Spatial persistence of fluctuating interfaces. Phys. Rev. Lett. 86, 3700-3703, 2001.

65. H. P. McKean. A winding problem for a resonator driven by a white noise. J. Math. Kyoto Univ. 2, 227-235, 1963.

66. R. Metzler, G. Oshanin, and S. Redner. First-passage Phenomena and their applications. World Scientific, 2014.

67. G. M. Molchan. Maximum of a fractional Brownian motion: Probabilities of small values. Comm. Math. Phys. 205 (1), 97-111, 1999. 
68. G. M. Molchan. Unilateral small deviations of processes related to the fractional Brownian motion. Stoch. Proc. Appl. 118 (11), 2085-2097, 2008.

69. G. M. Molchan. Survival exponents for some Gaussian processes. Int. J. Stoch. Anal. 2012, Article ID 137271, 2012.

70. G. M. Molchan and A. Khokhlov. Small values of the maximum for the integral of fractional Brownian motion. J. Stat. Phys. 114 (3-4), 923-946, 2004.

71. A. Novikov and N. Kordzakhia. Martingales and first passage times of AR(1) sequences. Stochastics 80, 197-210, 2008.

72. G. Oshanin, A. Rosso, G. Schehr. Anomalous fluctuations of currents in Sinai-type random chains with strongly correlated disorder. Phys. Rev. Lett. 110, 100602, 2013.

73. C. Profeta and T. Simon. Persistence of integrated stable processes. Preprint, arXiv:1403.1064, Probability Theory and Related Fields, to appear.

74. C. Profeta and T. Simon. Windings of the stable Kolmogorov process. Preprint, arXiv:1407.1597, ALEA Lat. Am. J. Probab. Math. Stat., to appear.

75. P. Revesz. Random walks in random and non-random environments. World Scientific, Singapore, 1990

76. G. Samorodnitsky and M. S. Taqqu. Stable non-Gaussian random processes. Chapman \& Hall; New-York, 1994

77. G. Schehr and S. N. Majumdar. Statistics of the Number of Zero Crossings: from Random Polynomials to the Diffusion Equation. Phys. Rev. Lett. 99, 603-606, 2007.

78. G. Schehr and S. N. Majumdar. Real roots of random polynomials and zero crossing properties of diffusion equation. J. Stat. Phys. 132 (2), 235-273, 2008.

79. Z.-S. She, E. Aurell and U. Frisch. The inviscid Burgers equation with initial data of Brownian type. Comm. Math. Phys. 148 (3), 623-641, 1992.

80. T. Simon. The lower tail problem for homogeneous functionals of stable processes with no negative jumps. ALEA Lat. Am. J. Probab. Math. Stat. 3, 165-179, 2007.

81. T. Simon. On the Hausdorff dimension of regular points of inviscid Burgers equation with stable initial data. J. Stat. Phys. 131 (4), 733-747, 2008.

82. Ya. G. Sinai. Distribution of some functionals of the integral of a random walk. Theor. Math. Phys. 90 (3), 219-241, 1992.

83. Ya. G. Sinai. Statistics of shocks in solutions of inviscid Burgers equation. Comm. Math. Phys. 148 (3), 601-621, 1992.

84. V. Vysotsky. Clustering in a stochastic model of one-dimensional gas. Ann. Appl. Probab. 18 (3), 1026-1058, 2008.

85. V. Vysotsky. On the probability that integrated random walks stay positive. Stoch. Proc. Appl. 120 (7), 1178-1193, 2010.

86. V. Vysotsky. Positivity of integrated random walks. To appear in Annales de l'Institut Henri Poincaré.

87. V. Vysotsky. Persistence of iterated random walks. Preprint, 2012.

88. E. Wong. Some results concerning the zero-crossings of Gaussian noise. SIAM J. Appl. Math. 14 (6), 1246-1254, 1966.

89. V. M. Zolotarev. One-dimensional stable distributions. AMS, Providence, 1986. 